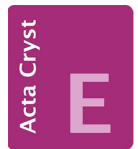

CRYSTALLOGRAPHIC COMMUNICATIONS

ISSN 2056-9890

Received 29 April 2015

Accepted 9 June 2015

Edited by G. Smith, Queensland University of Technology, Australia

Keywords: crystal structure; $\mathrm{Ba}-\mathrm{Zn}$ dinuclear complex; triphenylacetate ligand; 2-methoxyethanol; hydrogen bonding

CCDC reference: 1405801 Supporting information: this article has supporting information at journals.iucr.org/e

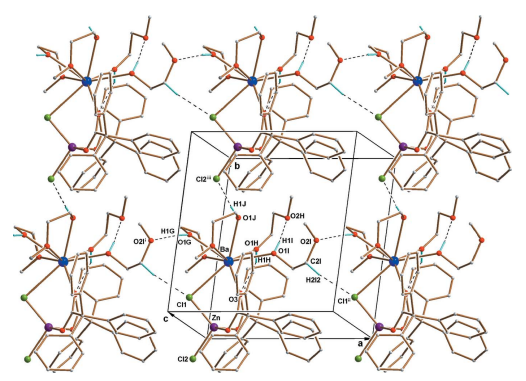

OPEN $\odot$ ACCESS

\section{Crystal structure of a mixed-ligand dinuclear Ba-Zn complex with 2-methoxyethanol having triphenylacetate and chloride bridges}

\author{
Józef Utko, ${ }^{a}$ Maria Sobocińska, ${ }^{a *}$ Danuta Dobrzyńska ${ }^{\mathrm{b}}$ and Tadeusz Lis ${ }^{\mathrm{a}}$
}

${ }^{\mathbf{a}}$ Faculty of Chemistry, University of Wrocław, 14 Joliot-Curie St, 50-383 Wrocław, Poland, and ${ }^{\mathbf{b}}$ Faculty of Chemistry, Wrocław University of Technology, 27 Wybrzeże Wyspiańskiego, 50-370 Wrocław, Poland. *Correspondence e-mail: maria.sobocinska@chem.uni.wroc.pl

The dinuclear barium-zinc complex, $\mu$-chlorido- $1: 2 \kappa^{2} \mathrm{Cl}$ :Cl-chlorido- $2 \kappa \mathrm{Cl}$-bis(2methoxyethanol- $1 \kappa O)$ bis $\left(2\right.$-methoxyethanol- $\left.1 \kappa^{2} O, O^{\prime}\right)$ bis $(\mu$-triphenylacetato1:2 $\left.\kappa^{2} O: O^{\prime}\right)$ bariumzinc, $\left[\mathrm{BaZn}\left(\mathrm{C}_{20} \mathrm{H}_{15} \mathrm{O}_{2}\right)_{2} \mathrm{Cl}_{2}\left(\mathrm{C}_{3} \mathrm{H}_{8} \mathrm{O}_{2}\right)_{4}\right]$, has been synthesized by the reaction of barium triphenylacetate, anhydrous zinc chloride and 2-methoxyethanol in the presence of toluene. The barium and zinc metal cations in the dinuclear complex are linked via one chloride anion and carboxylate $\mathrm{O}$ atoms of the triphenylacetate ligands, giving a $\mathrm{Ba} \cdots \mathrm{Zn}$ separation of 3.9335 (11) $\AA$. The irregular nine-coordinate $\mathrm{BaO}_{8} \mathrm{Cl}$ coordination centres comprise eight $\mathrm{O}$-atom donors, six of them from 2-methoxyethanol ligands (four from two bidentate $O, O^{\prime}$-chelate interactions and two from monodentate interactions), two from bridging triphenylacetate ligands and one from a bridging $\mathrm{Cl}$ donor. The distorted tetrahedral coordination sphere of zinc comprises two $\mathrm{O}$-atom donors from the triphenylacetate ligands and two $\mathrm{Cl}$ donors (one bridging and one terminal). In the crystal, $\mathrm{O}-\mathrm{H} \cdots \mathrm{Cl}, \mathrm{O}-\mathrm{H} \cdots \mathrm{O}$ and $\mathrm{C}-\mathrm{H} \cdots \mathrm{Cl}$ intermolecular interactions form a layered structure, lying parallel to (001).

\section{Chemical context}

Only a few polynuclear heterometallic compounds containing barium and zinc connected by carboxylate bridges are known (Akine et al., 2006, 2009, 2010; Zhang et al., 2012; Bo et al., 2013). We have been studying the reactions of the triphenylacetate anion with metal salts and we have obtained several anhydrous polynuclear $\mathrm{Mn}^{\mathrm{II}}$ triphenylacetate-containing clusters (Utko et al., 2014). The complexes with some metals (for example: $\mathrm{Fe}, \mathrm{Ni}, \mathrm{Cu}, \mathrm{Ru}, \mathrm{Rh}, \mathrm{Ag}$ ) are reported in the literature (Yamanaka et al., 1993; Cotton et al., 1994; Akhbari \& Morsali, 2010; Barberis et al., 2001; Cadiou et al., 2002; Do \& Lippard, 2011). However, among polynuclear complexes with triphenylacetate ligands, dinuclear $\mathrm{Ba}-\mathrm{Zn}$ representatives have not previously been reported. In the present work, we aimed to create a mixed-ligand compound containing zinc and barium cations, using barium triphenylacetate as a means of displacing chlorine atoms from zinc chloride. This procedure for removal of chlorine using triphenylacetate was successfully carried out in a reaction leading to the formation of a mixedmetal complex with a $\left[\mathrm{Ba}_{4} \mathrm{Ti}_{2}\right]$ core (Kosińska-Klähn et al., 2014). In the present paper we report the synthesis and structural characterization of a dinuclear $\mathrm{Ba}-\mathrm{Zn}$ complex, namely $\mu$-chlorido-1: $2 \kappa^{2} \mathrm{Cl}$ :Cl-chlorido- $2 \kappa \mathrm{Cl}$-bis(2-methoxyethanol-1 $\kappa O$ ) bis (2-methoxyethanol- $\left.1 \kappa^{2} O, O^{\prime}\right)$ bis $(\mu$-triphenyl-

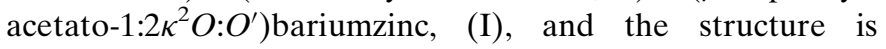
discussed herein. 


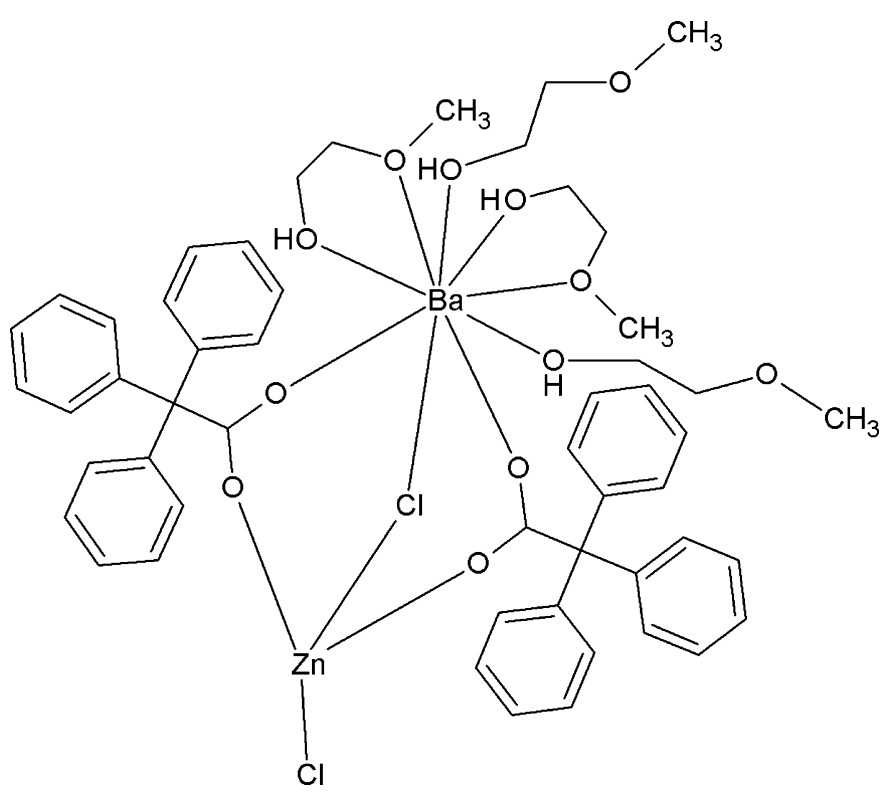

\section{Structural commentary}

In the structure of (I), the asymmetric unit contains one dinuclear complex of $\left[\mathrm{BaZn}\left(\mathrm{Ph}_{3} \mathrm{CCOO}\right)_{2}\left(\mathrm{CH}_{3} \mathrm{OCH}_{2}\right.\right.$ $\left.\left.\mathrm{CH}_{2} \mathrm{OH}\right)_{4} \mathrm{Cl}_{2}\right]$ (Fig. 1), in which the dinuclear $[\mathrm{BaZn}]^{4+}$ cationic core is bridged by two carboxylate arms of the triphenylacetate ligands in a $\kappa^{1}: \kappa^{1}: \mu^{2}$ coordination mode and by one bridging chlorine atom $\left(\mu_{2}-\mathrm{Cl}\right)$. The $\mathrm{Ba} \cdots \mathrm{Zn}$ distance in the dinuclear complex is 3.9335 (11) $\AA$. Oxygen atoms have the largest contribution to the filling of the coordination sphere of barium [ $\mathrm{Ba}-\mathrm{O}$ bond-length range, 2.6925 (19)2.985 (2) $\AA$; Table 1]. Barium is bonded to one bridging chloride atom $\left(\mu_{2}-\mathrm{Cl}\right)$, two $\mathrm{O}$-atoms of two carboxylate groups

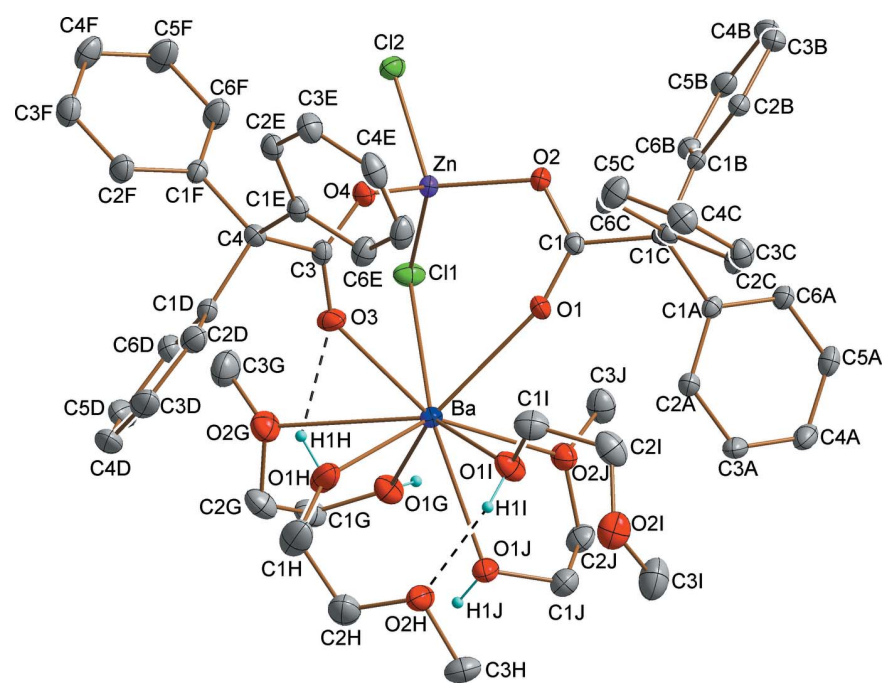

Figure 1

The molecular structure of the title complex, with displacement ellipsoids drawn at the $50 \%$ probability level. Dashed lines represent intra-complex hydrogen bonds. C-bonded $\mathrm{H}$ atoms have been omitted for clarity.
Table 1

Selected bond lengths $(\AA)$.

\begin{tabular}{llll}
\hline $\mathrm{Ba}-\mathrm{O} 3$ & $2.6925(19)$ & $\mathrm{Ba}-\mathrm{O} 2 G$ & $2.985(2)$ \\
$\mathrm{Ba}-\mathrm{O} 1$ & $2.7073(19)$ & $\mathrm{Ba}-\mathrm{Cl} 1$ & $3.1118(11)$ \\
$\mathrm{Ba}-\mathrm{O} 1 J$ & $2.7572(19)$ & $\mathrm{Zn}-\mathrm{O} 2$ & $1.9682(17)$ \\
$\mathrm{Ba}-\mathrm{O} 1 H$ & $2.783(2)$ & $\mathrm{Zn}-\mathrm{O} 4$ & $1.9683(18)$ \\
$\mathrm{Ba}-\mathrm{O} 2 J$ & $2.7908(19)$ & $\mathrm{Zn}-\mathrm{Cl} 1$ & $2.2595(10)$ \\
$\mathrm{Ba}-\mathrm{O} 1 G$ & $2.799(2)$ & $\mathrm{Zn}-\mathrm{Cl} 2$ & $2.2653(9)$ \\
$\mathrm{Ba}-\mathrm{O} 1 I$ & $2.810(2)$ & & \\
\hline
\end{tabular}

and also to six $\mathrm{O}$ atoms from the 2-methoxyethanol ligands (four from two bidentate $O, O^{1}$-chelate interactions and two from monodentate interactions). 2-Methoxyethanol is coordinated only to the $\mathrm{Ba}^{2+}$ cation. The coordination mode is achieved in two different ways. Two terminal molecules representing an $\kappa^{1}: \kappa^{1}$ mode form two five-membered rings completed by the barium atom. Two other molecules of 2methoxyethanol coordinate to Ba only through the hydroxyl $\mathrm{O}$ atoms.

Zinc is four-coordinated with a distorted tetrahedral $\mathrm{ZnO}_{2} \mathrm{Cl}_{2}$ stereochemistry $($ Table 1$)$, with $\mathrm{Zn}-\mathrm{Cl} 1$ (bridging) $=$ 2.2595 (10) $\AA$ and $\mathrm{Zn}-\mathrm{Cl} 2$ (monodentate) $=2.2653$ (9) $\AA$ and $\mathrm{Zn}-\mathrm{O}$ (both from the bridging triphenylacetate groups = 1.96817 (2) and $1.9683(18) \AA$ ). A comparison with other structurally characterized mixed-metallic zinc-barium complexes reveals that the $\mathrm{Zn}-\mathrm{Cl}-\mathrm{Ba}$ linkage has been observed for the first time in the present compound. There are only a few compounds containing both of these metals and only one is a dimeric structure, with a distance between the atoms of 3.629 (2) $\AA$, significantly shorter than in the title complex [3.9335 (11) $\AA$ ], but zinc and barium are connected only via bridging oxygen atoms $\left(\mu_{2}-\mathrm{O}\right)$ from organic ligands (Van Veggel et al., 1989). Also, in other structures without carboxylate bridges, the $\mathrm{Zn} \cdots \mathrm{Ba}$ distances are often much shorter than in the title complex with values in the range 3.4325 (5) to 4.850 (3) $\AA$ (Westerhausen et al., 2001, 2006; Baggio et al., 2004; John et al., 2008). In those cases where the oxygen atom $\left(\mu_{2}-\mathrm{O}\right)$ and also carboxylates connect zinc and barium, the $\mathrm{Zn} \cdots$ Ba distance is not longer than 3.638 (1) $\AA$ (Akine et al., 2006, 2009, 2010). In a polymeric structure where zinc and barium cations are bridged via two carboxylate arms and also via one molecule of water, the distance between them is 4.0208 (5) $\AA$ (Zhang et al., 2012).

\section{Supramolecular features}

In the crystal, there are intramolecular $\mathrm{O}-\mathrm{H} \cdots \mathrm{O}$ hydrogen bonds (Table 2). One is formed between a hydroxyl group O1I and an O-atom acceptor from the ether atom $(\mathrm{O} 2 \mathrm{H})$ of a 2-methoxyethanol ligand, the second is formed between a hydroxyl group $\mathrm{O} 1 \mathrm{H}$ and an O-atom acceptor from a carboxyl group (O3) of a $\mathrm{Ph}_{3} \mathrm{CCOO}^{-}$ligand (Fig. 1). The presence of electronegative atoms (oxygen and chlorine) also leads to the occurrence of intermolecular hydrogen bonds in the crystal structure. The neighbouring dinuclear molecules interact through $\mathrm{O}-\mathrm{H} \cdots \mathrm{O}, \mathrm{O}-\mathrm{H} \cdots \mathrm{Cl}$ and $\mathrm{C}-\mathrm{H} \cdots \mathrm{Cl}$ hydrogen 


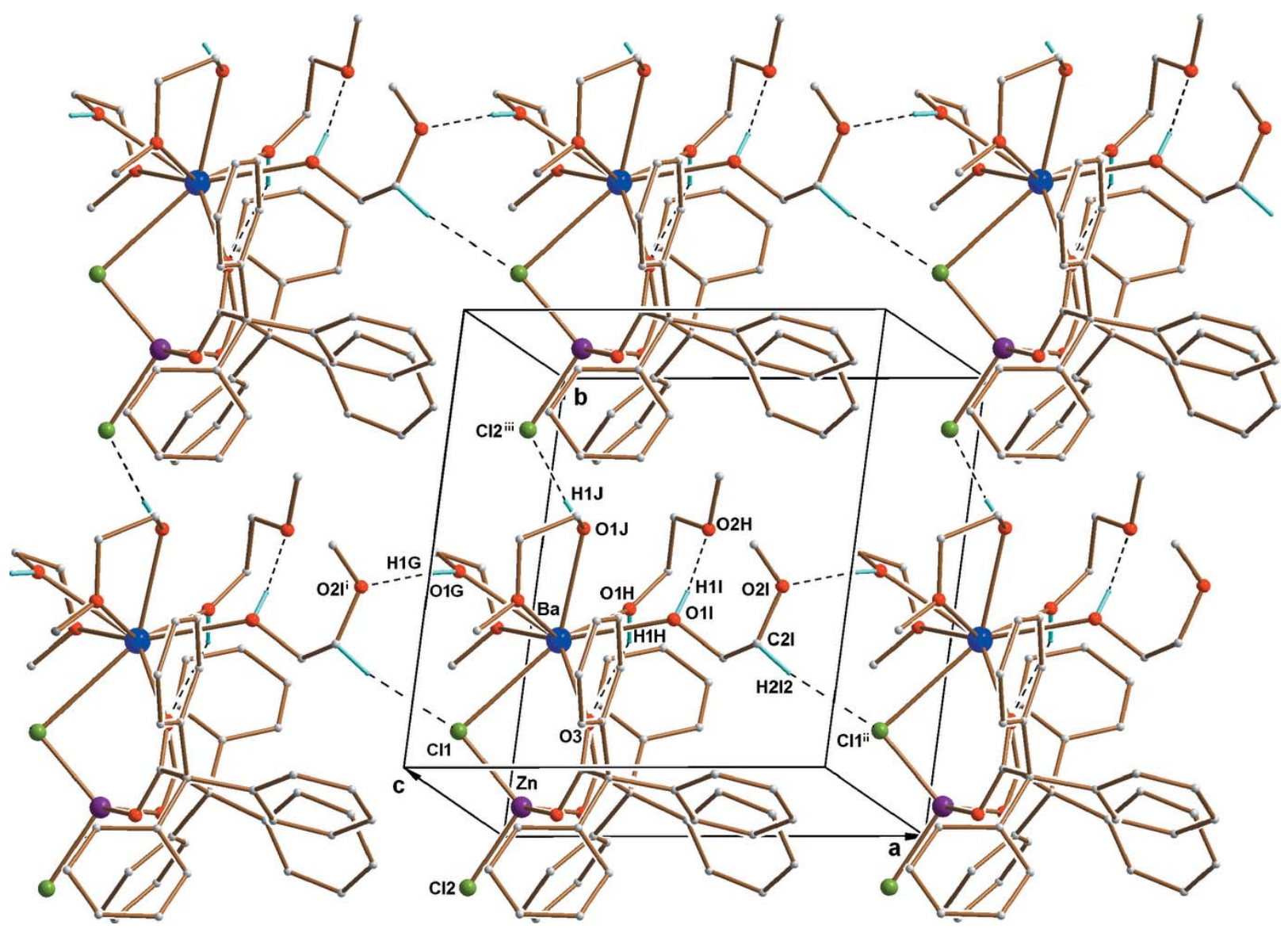

Figure 2

Part of the crystal structure of the complex. Dashed lines represent intra- and intermolecular hydrogen bonds. C-bonded $\mathrm{H}$ atoms not involved in hydrogen bonding have been omitted for clarity. For symmetry codes, see Table 2 .

bonds. The first one occurs between the hydroxyl group $\mathrm{O} 1 G$ and an ether $\mathrm{O}$-atom acceptor $\mathrm{O} 2 I^{\mathrm{i}}$, the second occurs between the hydroxyl group $\mathrm{O} 1 J$ and the terminal chlorine atom $\mathrm{Cl} 2{ }^{\mathrm{iii}}$. In the third interaction, the $\mathrm{H}$-donor atom is from a 2-methoxyethanol carbon $(\mathrm{C} 2 I)$, with the bridging chlorine atom $\left(\mathrm{Cl} 1 \mathrm{I}^{\mathrm{ii}}\right.$ acting as the $\mathrm{H}$-atom acceptor (for symmetry codes, see Table 2). A two-dimensional network structure is generated (Fig. 2), lying parallel to (001).

\section{Synthesis and crystallization}

For the preparation of $\mathrm{Ba}\left(\mathrm{Ph}_{3} \mathrm{CCOO}\right)_{2}$, a mixture of metallic barium $(0.521 \mathrm{~g}, 3.8 \mathrm{mmol})$, triphenylacetic acid $(2.209 \mathrm{~g}$, $7.66 \mathrm{mmol}), \mathrm{C}_{6} \mathrm{H}_{5} \mathrm{CH}_{3}(50 \mathrm{ml})$ and THF $(10 \mathrm{ml})$ was stirred at $363-373 \mathrm{~K}$ for $24 \mathrm{~h}$ until all the metal had reacted. The solu-

Table 2

Hydrogen-bond geometry $\left(\AA,^{\circ}\right)$.

\begin{tabular}{lllll}
\hline$D-\mathrm{H} \cdots A$ & $D-\mathrm{H}$ & $\mathrm{H} \cdots A$ & $D \cdots A$ & $D-\mathrm{H} \cdots A$ \\
\hline $\mathrm{O} 1 G-\mathrm{H} 1 G \cdots \mathrm{O} 2 I^{\mathrm{i}}$ & 0.84 & 1.91 & $2.728(3)$ & 163 \\
$\mathrm{O} 1 I-\mathrm{H} 1 I \cdots \mathrm{O} 2 H$ & 0.84 & 1.99 & $2.817(3)$ & 170 \\
$\mathrm{C} 2 I-\mathrm{H} 2 I 2 \cdots \mathrm{Cl} 1^{\mathrm{ii}}$ & 0.99 & 2.81 & $3.660(3)$ & 144 \\
$\mathrm{O} 1 J-\mathrm{H} 1 J \cdots \mathrm{C} 2^{\mathrm{iii}}$ & 0.84 & 2.17 & $3.012(2)$ & 174 \\
\hline
\end{tabular}

Symmetry codes: (i) $x-1, y, z$; (ii) $x+1, y, z$; (iii) $x, y+1, z$. tion, which included a white precipitate, was concentrated to about $20 \mathrm{ml}$ and then hexane $(50 \mathrm{ml})$ was added while stirring, which led to further precipitation. The product was filtered on a Schlenk flask (yield: $2.520 \mathrm{~g}, 93.26 \%$ ). Elemental analysis (\%) calculated for $\mathrm{Ba}\left(\mathrm{Ph}_{3} \mathrm{CCOO}\right)_{2}$ : C 67.48, H 5.38, Ba 19.29; found: $\mathrm{C}$ 67.56, $\mathrm{H}$ 5.51, Ba 19.44. Solid $\mathrm{ZnCl}_{2}(0.273 \mathrm{~g}$, $2.0 \mathrm{mmol})$ and $\mathrm{Ba}\left(\mathrm{Ph}_{3} \mathrm{CCOO}\right)_{2}(1.426 \mathrm{~g}, 2.0 \mathrm{mmol})$ were then added to a solution of $\mathrm{CH}_{3} \mathrm{OCH}_{2} \mathrm{CH}_{2} \mathrm{OH}(30 \mathrm{ml})$ and $\mathrm{C}_{6} \mathrm{H}_{5} \mathrm{CH}_{3}(15 \mathrm{ml})$ and the resulting mixture was stirred under a nitrogen atmosphere for $24 \mathrm{~h}$. The solution was filtered and then concentrated to about $20 \mathrm{ml}$. Afterwards $20 \mathrm{ml}$ of hexane was funneled into the reaction solution, leading to the creation of two layers and the mixture was left to crystallize at room temperature. After one week, colorless crystals suitable for the X-ray experiment were obtained $(1.289 \mathrm{~g}$, yield: $55.83 \%)$. Knowledge of the molecular structure of the final product enables representation of the chemical equation for the reaction as:

\section{$\mathrm{ZnCl}_{2}+\mathrm{Ba}\left(\left(\mathrm{C}_{6} \mathrm{H}_{5}\right)_{3} \mathrm{CCOO}\right)_{2}+4\left(\mathrm{CH}_{3} \mathrm{OCH}_{2} \mathrm{CH}_{2} \mathrm{OH}\right) \rightarrow$ $\left[\mathrm{BaZnCl}_{2}\left[\left(\mathrm{C}_{6} \mathrm{H}_{5}\right)_{3} \mathrm{CCOO}\right]_{2}\left(\mathrm{CH}_{3} \mathrm{OCH}_{2} \mathrm{CH}_{2} \mathrm{OH}\right)_{4}\right]$.}

Elemental analysis: (\%) calculated for the complex: C 54.14, H 5.38, Cl 6.3, Zn 5.67, Ba 11.91; found: C 52.94, H 5.67, Zn 5.48, Ba 11.24. 
Table 3

Experimental details.

\begin{tabular}{|c|c|}
\hline \multicolumn{2}{|l|}{ Crystal data } \\
\hline Chemical formula & {$\left[\mathrm{BaZn}\left(\mathrm{C}_{20} \mathrm{H}_{15} \mathrm{O}_{2}\right)_{2} \mathrm{Cl}_{2}\left(\mathrm{C}_{3} \mathrm{H}_{8} \mathrm{O}_{2}\right)_{4}\right]$} \\
\hline$M_{\mathrm{r}}$ & 1152.62 \\
\hline Crystal system, space group & Triclinic, $P \overline{1}$ \\
\hline Temperature (K) & 100 \\
\hline$a, b, c(\AA)$ & $9.706(3), 10.643(3), 25.073(6)$ \\
\hline$\alpha, \beta, \gamma\left({ }^{\circ}\right)$ & $89.62(3), 89.26(3), 82.73(3)$ \\
\hline$V\left(\AA^{3}\right)$ & $2569.0(12)$ \\
\hline$Z$ & 2 \\
\hline Radiation type & Mo $K \alpha$ \\
\hline$\mu\left(\mathrm{mm}^{-1}\right)$ & 1.39 \\
\hline Crystal size $(\mathrm{mm})$ & $0.31 \times 0.23 \times 0.21$ \\
\hline \multicolumn{2}{|l|}{ Data collection } \\
\hline Diffractometer & Oxford Diffraction KM-4-CCD \\
\hline Absorption correction & $\begin{array}{l}\text { Analytical [CrysAlis RED (Oxford } \\
\text { Diffraction, 2010), based on } \\
\text { expressions derived by Clark \& } \\
\text { Reid (1995)] }\end{array}$ \\
\hline$T_{\min }, T_{\max }$ & $0.687,0.780$ \\
\hline $\begin{array}{l}\text { No. of measured, independent and } \\
\text { observed }[I>2 \sigma(I)] \text { reflections }\end{array}$ & $24098,12296,10742$ \\
\hline$R_{\text {int }}$ & 0.025 \\
\hline$(\sin \theta / \lambda)_{\max }\left(\AA^{-1}\right)$ & 0.705 \\
\hline \multicolumn{2}{|l|}{ Refinement } \\
\hline$R\left[F^{2}>2 \sigma\left(F^{2}\right)\right], w R\left(F^{2}\right), S$ & $0.035,0.100,1.14$ \\
\hline No. of reflections & 12296 \\
\hline No. of parameters & 617 \\
\hline $\mathrm{H}$-atom treatment & $\mathrm{H}$-atom parameters constrained \\
\hline$\Delta \rho_{\max }, \Delta \rho_{\min }\left(\mathrm{e} \AA^{-3}\right)$ & $0.89,-0.57$ \\
\hline
\end{tabular}

Computer programs: CrysAlis CCD and CrysAlis RED (Oxford Diffraction, 2010), SHELXS97 and SHELXTL (Sheldrick, 2008) and SHELXL2013 (Sheldrick, 2015).

\section{Refinement details}

Crystal data, data collection and structure refinement details are summarized in Table 3. All $\mathrm{C}$-bonded $\mathrm{H}$ atoms were positioned geometrically and treated as riding atoms: methyl $\mathrm{H}$ atoms were constrained to an ideal geometry, with $\mathrm{C}-\mathrm{H}=$ $0.98 \AA$ and $U_{\text {iso }}(\mathrm{H})=1.5 U_{\text {eq }}(\mathrm{C})$; the remaining $\mathrm{H}$ atoms were afixed to $\mathrm{C}$ atoms, with $\mathrm{Csp} p^{2}-\mathrm{H}=0.95 \AA$ and $\mathrm{Csp} p^{3}-\mathrm{H}=$ $0.99 \AA$, and with $U_{\text {iso }}(\mathrm{H})=1.2 U_{\text {eq }}(\mathrm{C})$. The locations of $\mathrm{H}$ atoms of the hydroxyl groups were determined from a difference-Fourier map and finally constrained to ride on their parent atoms, with $\mathrm{O}-\mathrm{H}=0.84 \AA$ and $U_{\text {iso }}(\mathrm{H})=$ $1.5 U_{\mathrm{eq}}(\mathrm{O})$.

\section{References}

Akhbari, K. \& Morsali, A. (2010). CrystEngComm, 12, 3394-3396. Akine, S., Kagiyama, S. \& Nabeshima, T. (2010). Inorg. Chem. 49, 2141-2152.

Akine, S., Morita, Y., Utsuno, F. \& Nabeshima, T. (2009). Inorg. Chem. 48, 10670-10678.

Akine, S., Taniguchi, T. \& Nabeshima, T. (2006). J. Am. Chem. Soc. 128, 15765-15774.

Baggio, R., Stoilova, D., Polla, G., Leyva, G. \& Garland, M. T. (2004). J. Mol. Struct. 697, 173-180.

Barberis, M., Lahuerta, P., Perez-Prieto, J. \& Sanau, M. (2001). Chem. Commun. pp. 439-440.

Bo, Q.-B., Wang, H.-Y. \& Wang, D.-Q. (2013). New J. Chem. 37, 380390.

Cadiou, C., Coxal, R. A., Graham, A., Harisson, A., Helliwell, M., Parsons, S. \& Winpenny, R. R. P. (2002). Chem. Commun. pp. 11061107.

Clark, R. C. \& Reid, J. S. (1995). Acta Cryst. A51, 887-897.

Cotton, F. A., Daniels, L. M., Kibala, P. A., Matusz, M., Roth, W. J., Schwotzer, W., Wenning, W. \& Bianxiao, Z. (1994). Inorg. Chim. Acta, 215, 9-15.

Do, L. H. \& Lippard, S. J. (2011). J. Am. Chem. Soc. 133, 1056810581.

John, Ł., Utko, J., Szafert, S., Jerzykiewicz, L. B., Kepiński, L. \& Sobota, P. (2008). Chem. Mater. 20, 4231-4239.

Kosińska-Klähn, M., Łukasz, J., Drąg-Jarząbek, A., Utko, J., Petrus, R., Jerzykiewicz, L. B. \& Sobota, P. (2014). Inorg. Chem. 53, 16301636.

Oxford Diffraction (2010). CrysAlis CCD and CrysAlis RED. Oxford Diffraction Ltd., Yarnton, England.

Sheldrick, G. M. (2008). Acta Cryst. A64, 112-122.

Sheldrick, G. M. (2015). Acta Cryst. C71, 3-8.

Utko, J., Canaj, A. B., Milios, C. J., Dobrzyńska, D., Pawlus, K., Mikołajczyk, A. \& Lis, T. (2014). Inorg. Chim. Acta, 409, 458-464.

Van Veggel, F. C. J. M., Harkema, S., Bos, M., Verboom, W., Van Staveren, C. J., Gerritsma, G. J. \& Reinhoudt, D. N. (1989). Inorg. Chem. 28, 1133-1148.

Westerhausen, M., Gückel, C., Habereder, T., Vogt, M., Warchhold, M. \& Nöth, H. (2001). Organometallics, 20, 893-899.

Westerhausen, M., Sapelza, G. \& Mayer, P. (2006). Inorg. Chem. Commun. 9, 949-951.

Yamanaka, M., Ohba, S., Tokii, T., Jury, C. F., Steward, O. W. \& Kato, M. (1993). Acta Cryst. C49, 1469-1473.

Zhang, X., Huang, Y.-Y., Cheng, J.-K., Yao, Y.-G., Zhang, J. \& Wang, F. (2012). CrystEngComm, 14, 4843-4849. 


\section{supporting information}

Acta Cryst. (2015). E71, 791-794 [doi:10.1107/S2056989015011226]

\section{Crystal structure of a mixed-ligand dinuclear Ba-Zn complex with 2-methoxy- ethanol having triphenylacetate and chloride bridges}

\section{Józef Utko, Maria Sobocińska, Danuta Dobrzyńska and Tadeusz Lis}

\section{Computing details}

Data collection: CrysAlis CCD (Oxford Diffraction, 2010); cell refinement: CrysAlis CCD (Oxford Diffraction, 2010); data reduction: CrysAlis RED (Oxford Diffraction, 2010); program(s) used to solve structure: SHELXS97 (Sheldrick, 2008); program(s) used to refine structure: SHELXL2013 (Sheldrick, 2015); molecular graphics: SHELXTL (Sheldrick, 2008); software used to prepare material for publication: SHELXL2013 (Sheldrick, 2015).

$\mu$-Chlorido-1:2 $\kappa^{2} \mathrm{Cl}$ :Cl-chlorido-2 $\kappa \mathrm{Cl}$-bis(2-methoxyethanol-1 $\left.\kappa O\right)$ bis(2-methoxyethanol-1 $\left.\kappa^{2} \mathrm{O}, \mathrm{O}^{\prime}\right) \mathrm{bis}(\mu$ triphenylacetato-1:2 $\left.\kappa^{2} O: O^{\prime}\right)$ bariumzinc

Crystal data

$\left[\mathrm{BaZn}\left(\mathrm{C}_{20} \mathrm{H}_{15} \mathrm{O}_{2}\right)_{2} \mathrm{Cl}_{2}\left(\mathrm{C}_{3} \mathrm{H}_{8} \mathrm{O}_{2}\right)_{4}\right]$

$M_{r}=1152.62$

Triclinic, $P \overline{1}$

$a=9.706(3) \AA$

$b=10.643(3) \AA$

$c=25.073(6) \AA$

$\alpha=89.62(3)^{\circ}$

$\beta=89.26(3)^{\circ}$

$\gamma=82.73(3)^{\circ}$

$V=2569.0(12) \AA^{3}$

Data collection

Oxford Diffraction KM-4-CCD diffractometer

Radiation source: fine-focus sealed tube $\omega$ scans

Absorption correction: analytical

[CrysAlis RED (Oxford Diffraction, 2010), based on expressions derived by Clark \& Reid (1995)]

$T_{\min }=0.687, T_{\max }=0.780$

Refinement

Refinement on $F^{2}$

Least-squares matrix: full

$R\left[F^{2}>2 \sigma\left(F^{2}\right)\right]=0.035$

$w R\left(F^{2}\right)=0.100$

$S=1.14$

12296 reflections

617 parameters
$Z=2$

$F(000)=1180$

$D_{\mathrm{x}}=1.490 \mathrm{Mg} \mathrm{m}^{-3}$

Mo $K \alpha$ radiation, $\lambda=0.71073 \AA$

Cell parameters from 17769 reflections

$\theta=2-31^{\circ}$

$\mu=1.39 \mathrm{~mm}^{-1}$

$T=100 \mathrm{~K}$

Block, colorless

$0.31 \times 0.23 \times 0.21 \mathrm{~mm}$

24098 measured reflections 12296 independent reflections 10742 reflections with $I>2 \sigma(I)$

$R_{\text {int }}=0.025$

$\theta_{\max }=30.1^{\circ}, \theta_{\min }=2.8^{\circ}$

$h=-12 \rightarrow 12$

$k=-13 \rightarrow 13$

$l=-33 \rightarrow 35$

0 restraints

Primary atom site location: structure-invariant direct methods

Secondary atom site location: difference Fourier map

Hydrogen site location: inferred from neighbouring sites 
$\mathrm{H}$-atom parameters constrained

$w=1 /\left[\sigma^{2}\left(F_{\mathrm{o}}{ }^{2}\right)+(0.064 P)^{2}\right]$

where $P=\left(F_{\mathrm{o}}^{2}+2 F_{\mathrm{c}}^{2}\right) / 3$

$$
\begin{aligned}
& (\Delta / \sigma)_{\max }=0.001 \\
& \Delta \rho_{\max }=0.89 \mathrm{e}^{-3} \\
& \Delta \rho_{\min }=-0.57 \mathrm{e} \AA^{-3}
\end{aligned}
$$

\section{Special details}

Experimental. The O-bonded $\mathrm{H}$ atoms were found from a difference-Fourier map. These $\mathrm{H}$ atoms were included in the refinement with constraint:;finally with instruction Afix 3.

Absorption correction: CrysAlis RED (Oxford Diffraction, 2010), employing an analytical numeric absorption correction using a multifaceted crystal model based on expressions derived by R.C. Clark \& J.S. Reid (Clark \& Reid, 1995).

Geometry. All e.s.d.'s (except the e.s.d. in the dihedral angle between two 1.s. planes) are estimated using the full covariance matrix. The cell e.s.d.'s are taken into account individually in the estimation of e.s.d.'s in distances, angles and torsion angles; correlations between e.s.d.'s in cell parameters are only used when they are defined by crystal symmetry. An approximate (isotropic) treatment of cell e.s.d.'s is used for estimating e.s.d.'s involving 1.s. planes.

Refinement. Refinement of $F^{2}$ against ALL reflections. The weighted $R$-factor $w R$ and goodness of fit $S$ are based on $F^{2}$, conventional $R$-factors $R$ are based on $F$, with $F$ set to zero for negative $F^{2}$. The threshold expression of $F^{2}>\sigma\left(F^{2}\right)$ is used only for calculating $R$-factors(gt) etc. and is not relevant to the choice of reflections for refinement. $R$-factors based on $F^{2}$

\begin{tabular}{|c|c|c|c|c|}
\hline & $x$ & $y$ & $z$ & $U_{\text {iso }} * / U_{\text {eq }}$ \\
\hline $\mathrm{Ba}$ & $0.13461(2)$ & $0.39066(2)$ & $0.25149(2)$ & $0.01587(5)$ \\
\hline $\mathrm{Zn}$ & 0.09859 & $0.02666(2)$ & $0.25185(2)$ & $0.01406(7)$ \\
\hline $\mathrm{C} 11$ & $-0.07279(6)$ & $0.19070(6)$ & $0.25860(3)$ & $0.02295(13)$ \\
\hline $\mathrm{Cl} 2$ & $-0.00737(6)$ & $-0.14768(6)$ & $0.23751(2)$ & $0.02093(12)$ \\
\hline $\mathrm{O} 1$ & 0.24715 (19) & $0.19812(16)$ & $0.31457(7)$ & $0.0202(4)$ \\
\hline $\mathrm{O} 2$ & $0.20140(18)$ & $-0.00059(16)$ & $0.31895(6)$ & $0.0178(3)$ \\
\hline $\mathrm{C} 1$ & $0.2527(2)$ & $0.0946(2)$ & $0.33730(9)$ & $0.0147(4)$ \\
\hline $\mathrm{C} 2$ & $0.3316(2)$ & $0.0704(2)$ & $0.39131(9)$ & $0.0131(4)$ \\
\hline C1A & $0.3078(2)$ & $0.1863(2)$ & $0.42871(9)$ & $0.0141(4)$ \\
\hline $\mathrm{C} 2 \mathrm{~A}$ & 0.3301 & $0.3070(2)$ & $0.41065(9)$ & $0.0196(5)$ \\
\hline $\mathrm{H} 2 \mathrm{~A}$ & 0.3540 & 0.3190 & 0.3743 & $0.024 *$ \\
\hline C3A & 0.3179 & $0.4090(2)$ & $0.44516(10)$ & $0.0238(5)$ \\
\hline H3A & 0.3340 & 0.4900 & 0.4321 & $0.029 *$ \\
\hline $\mathrm{C} 4 \mathrm{~A}$ & $0.2824(3)$ & $0.3948(2)$ & $0.49867(10)$ & $0.0247(5)$ \\
\hline H4A & 0.2729 & 0.4654 & 0.5220 & $0.030 *$ \\
\hline C5A & 0.2613 & $0.2763(2)$ & $0.51715(10)$ & $0.0222(5)$ \\
\hline H5A & 0.2372 & 0.2648 & 0.5535 & $0.027 *$ \\
\hline C6A & $0.2752(3)$ & $0.1736(2)$ & $0.48271(9)$ & $0.0180(5)$ \\
\hline H6A & 0.2621 & 0.0923 & 0.4963 & $0.022 *$ \\
\hline C1B & $0.2833(2)$ & $-0.0447(2)$ & $0.41952(9)$ & $0.0148(4)$ \\
\hline $\mathrm{C} 2 \mathrm{~B}$ & $0.3746(3)$ & $-0.1460(2)$ & $0.43841(9)$ & $0.0175(5)$ \\
\hline $\mathrm{H} 2 \mathrm{~B}$ & 0.4712 & -0.1488 & 0.4311 & $0.021 *$ \\
\hline $\mathrm{C} 3 \mathrm{~B}$ & 0.3268 & $-0.2436(2)$ & $0.46791(10)$ & $0.0217(5)$ \\
\hline H3B & 0.3907 & -0.3120 & 0.4805 & $0.026^{*}$ \\
\hline $\mathrm{C} 4 \mathrm{~B}$ & $0.1867(3)$ & $-0.2410(2)$ & $0.47891(10)$ & $0.0233(5)$ \\
\hline H4B & 0.1543 & -0.3071 & 0.4993 & $0.028 *$ \\
\hline C5B & 0.0933 & $-0.1414(2)$ & $0.46006(10)$ & $0.0208(5)$ \\
\hline H5B & -0.0033 & -0.1393 & 0.4673 & $0.025^{*}$ \\
\hline
\end{tabular}
are statistically about twice as large as those based on $F$, and $R$ - factors based on ALL data will be even larger.

Fractional atomic coordinates and isotropic or equivalent isotropic displacement parameters $\left(\AA^{2}\right)$ 


\begin{tabular}{|c|c|c|c|c|}
\hline C6B & $0.1418(3)$ & $-0.0450(2)$ & $0.43054(9)$ & $0.0177(5)$ \\
\hline H6B & 0.0773 & 0.0224 & 0.4175 & $0.021^{*}$ \\
\hline $\mathrm{C} 1 \mathrm{C}$ & $0.4882(2)$ & $0.0448(2)$ & $0.37655(9)$ & $0.0144(4)$ \\
\hline $\mathrm{C} 2 \mathrm{C}$ & $0.5884(3)$ & $0.0737(2)$ & $0.41205(9)$ & $0.0187(5)$ \\
\hline $\mathrm{H} 2 \mathrm{C}$ & 0.5599 & 0.1150 & 0.4446 & $0.022 *$ \\
\hline $\mathrm{C} 3 \mathrm{C}$ & $0.7289(3)$ & $0.0433(2)$ & $0.40100(10)$ & $0.0216(5)$ \\
\hline $\mathrm{H} 3 \mathrm{C}$ & 0.7955 & 0.0634 & 0.4259 & $0.026^{*}$ \\
\hline $\mathrm{C} 4 \mathrm{C}$ & $0.7723(3)$ & $-0.0164(3)$ & $0.35348(10)$ & $0.0233(5)$ \\
\hline $\mathrm{H} 4 \mathrm{C}$ & 0.8684 & -0.0380 & 0.3458 & $0.028 *$ \\
\hline $\mathrm{C} 5 \mathrm{C}$ & $0.6735(3)$ & $-0.0441(3)$ & $0.31737(10)$ & $0.0256(6)$ \\
\hline $\mathrm{H} 5 \mathrm{C}$ & 0.7023 & -0.0844 & 0.2847 & $0.031^{*}$ \\
\hline $\mathrm{C} 6 \mathrm{C}$ & $0.5329(3)$ & $-0.0136(2)$ & $0.32859(10)$ & $0.0203(5)$ \\
\hline $\mathrm{H} 6 \mathrm{C}$ & 0.4664 & -0.0327 & 0.3034 & $0.024 *$ \\
\hline $\mathrm{O} 3$ & $0.2094(2)$ & $0.22740(16)$ & $0.17158(7)$ & $0.0244(4)$ \\
\hline $\mathrm{O} 4$ & $0.22946(18)$ & $0.02259(16)$ & $0.19108(6)$ & 0.0195 \\
\hline $\mathrm{C} 3$ & $0.2431(3)$ & $0.1152(2)$ & $0.16032(9)$ & $0.0167(5)$ \\
\hline $\mathrm{C} 4$ & $0.3131(2)$ & $0.0801(2)$ & $0.10490(9)$ & $0.0164(4)$ \\
\hline C1D & $0.3201(3)$ & $0.2003(2)$ & $0.07098(9)$ & $0.0189(5)$ \\
\hline $\mathrm{C} 2 \mathrm{D}$ & $0.4423(3)$ & $0.2304(3)$ & $0.04765(10)$ & $0.0245(5)$ \\
\hline $\mathrm{H} 2 \mathrm{D}$ & 0.5275 & 0.1794 & 0.0551 & $0.029^{*}$ \\
\hline C3D & 0.4417 (4) & $0.3342(3)$ & 0.01355 (11) & $0.0335(7)$ \\
\hline H3D & 0.5264 & 0.3524 & -0.0024 & $0.040^{*}$ \\
\hline C4D & $0.3199(4)$ & $0.4111(3)$ & $0.00251(12)$ & $0.0355(7)$ \\
\hline H4D & 0.3198 & 0.4817 & -0.0208 & $0.043^{*}$ \\
\hline C5D & 0.1979 (4) & $0.3830(3)$ & $0.02615(12)$ & $0.0356(7)$ \\
\hline H5D & 0.1133 & 0.4356 & 0.0194 & $0.043^{*}$ \\
\hline C6D & $0.1978(3)$ & $0.2785(3)$ & $0.05973(11)$ & $0.0253(5)$ \\
\hline H6D & 0.1128 & 0.2601 & 0.0753 & $0.030^{*}$ \\
\hline $\mathrm{C} 1 \mathrm{E}$ & $0.4604(2)$ & $0.0178(2)$ & $0.11792(9)$ & $0.0175(5)$ \\
\hline $\mathrm{C} 2 \mathrm{E}$ & $0.5232(3)$ & $-0.0894(2)$ & $0.09188(9)$ & $0.0201(5)$ \\
\hline $\mathrm{H} 2 \mathrm{E}$ & 0.4725 & -0.1297 & 0.0663 & $0.024 *$ \\
\hline $\mathrm{C} 3 \mathrm{E}$ & $0.6595(3)$ & $-0.1387(3)$ & $0.10280(10)$ & $0.0248(5)$ \\
\hline $\mathrm{H} 3 \mathrm{E}$ & 0.7010 & -0.2120 & 0.0845 & $0.030^{*}$ \\
\hline $\mathrm{C} 4 \mathrm{E}$ & $0.7351(3)$ & $-0.0822(3)$ & $0.13997(11)$ & $0.0281(6)$ \\
\hline $\mathrm{H} 4 \mathrm{E}$ & 0.8285 & -0.1159 & 0.1470 & $0.034 *$ \\
\hline $\mathrm{C} 5 \mathrm{E}$ & $0.6736(3)$ & $0.0245(3)$ & $0.16711(11)$ & $0.0283(6)$ \\
\hline $\mathrm{H} 5 \mathrm{E}$ & 0.7244 & 0.0634 & 0.1931 & $0.034 *$ \\
\hline C6E & $0.5379(3)$ & $0.0737(3)$ & $0.15608(10)$ & $0.0248(5)$ \\
\hline H6E & 0.4964 & 0.1466 & 0.1747 & $0.030^{*}$ \\
\hline $\mathrm{C} 1 \mathrm{~F}$ & $0.2325(2)$ & $-0.0103(2)$ & $0.07292(9)$ & $0.0167(5)$ \\
\hline $\mathrm{C} 2 \mathrm{~F}$ & $0.2503(3)$ & $-0.0153(3)$ & $0.01745(10)$ & $0.0249(5)$ \\
\hline $\mathrm{H} 2 \mathrm{~F}$ & 0.3067 & 0.0402 & 0.0005 & $0.030^{*}$ \\
\hline $\mathrm{C} 3 \mathrm{~F}$ & $0.1875(3)$ & $-0.0990(3)$ & $-0.01334(10)$ & $0.0277(6)$ \\
\hline $\mathrm{H} 3 \mathrm{~F}$ & 0.2015 & -0.1000 & -0.0509 & $0.033^{*}$ \\
\hline $\mathrm{C} 4 \mathrm{~F}$ & $0.1055(3)$ & $-0.1806(3)$ & $0.00986(11)$ & $0.0296(6)$ \\
\hline $\mathrm{H} 4 \mathrm{~F}$ & 0.0646 & -0.2398 & -0.0110 & $0.036^{*}$ \\
\hline $\mathrm{C} 5 \mathrm{~F}$ & $0.0841(3)$ & $-0.1744(3)$ & $0.06440(12)$ & $0.0351(7)$ \\
\hline $\mathrm{H} 5 \mathrm{~F}$ & 0.0253 & -0.2285 & 0.0809 & $0.042 *$ \\
\hline
\end{tabular}




\begin{tabular}{|c|c|c|c|c|}
\hline $\mathrm{C} 6 \mathrm{~F}$ & $0.1463(3)$ & $-0.0909(3)$ & $0.09569(11)$ & $0.0279(6)$ \\
\hline $\mathrm{H} 6 \mathrm{~F}$ & 0.1298 & -0.0890 & 0.1331 & $0.034^{*}$ \\
\hline $\mathrm{O} 1 \mathrm{G}$ & $-0.12372(19)$ & $0.54119(18)$ & $0.25180(8)$ & $0.0257(4)$ \\
\hline $\mathrm{H} 1 \mathrm{G}$ & -0.1790 & 0.5348 & 0.2774 & $0.039 *$ \\
\hline $\mathrm{C} 1 \mathrm{G}$ & $-0.1947(3)$ & $0.5900(3)$ & $0.20479(11)$ & $0.0276(6)$ \\
\hline H1G1 & -0.2228 & 0.6823 & 0.2081 & $0.033^{*}$ \\
\hline $\mathrm{H} 1 \mathrm{G} 2$ & -0.2792 & 0.5484 & 0.1996 & $0.033^{*}$ \\
\hline $\mathrm{C} 2 \mathrm{G}$ & $-0.0963(3)$ & $0.5630(3)$ & $0.15848(12)$ & $0.0299(6)$ \\
\hline $\mathrm{H} 2 \mathrm{G} 1$ & -0.1454 & 0.5896 & 0.1251 & $0.036^{*}$ \\
\hline $\mathrm{H} 2 \mathrm{G} 2$ & -0.0186 & 0.6142 & 0.1621 & $0.036^{*}$ \\
\hline $\mathrm{O} 2 \mathrm{G}$ & $-0.0411(2)$ & $0.43219(19)$ & $0.15458(8)$ & $0.0293(4)$ \\
\hline $\mathrm{C} 3 \mathrm{G}$ & $-0.1399(4)$ & $0.3533(3)$ & $0.13765(13)$ & $0.0390(7)$ \\
\hline $\mathrm{H} 3 \mathrm{G} 1$ & -0.2184 & 0.3603 & 0.1629 & $0.058^{*}$ \\
\hline H3G2 & -0.0962 & 0.2652 & 0.1363 & $0.058^{*}$ \\
\hline H3G3 & -0.1732 & 0.3802 & 0.1021 & $0.058^{*}$ \\
\hline $\mathrm{O} 1 \mathrm{H}$ & $0.2695(2)$ & $0.4717(2)$ & $0.16137(8)$ & $0.0352(5)$ \\
\hline $\mathrm{H} 1 \mathrm{H}$ & 0.2743 & 0.4048 & 0.1435 & $0.053^{*}$ \\
\hline $\mathrm{C} 1 \mathrm{H}$ & $0.3414(4)$ & $0.5586(3)$ & $0.13328(13)$ & $0.0425(8)$ \\
\hline H1H1 & 0.2929 & 0.5839 & 0.0996 & $0.051^{*}$ \\
\hline $\mathrm{H} 1 \mathrm{H} 2$ & 0.4365 & 0.5188 & 0.1243 & $0.051^{*}$ \\
\hline $\mathrm{C} 2 \mathrm{H}$ & $0.3480(4)$ & $0.6720(3)$ & $0.16707(13)$ & $0.0366(7)$ \\
\hline $\mathrm{H} 2 \mathrm{H} 1$ & 0.3783 & 0.7415 & 0.1452 & $0.044 *$ \\
\hline $\mathrm{H} 2 \mathrm{H} 2$ & 0.2549 & 0.7013 & 0.1823 & $0.044^{*}$ \\
\hline $\mathrm{O} 2 \mathrm{H}$ & $0.4452(2)$ & $0.63873(17)$ & $0.20935(8)$ & 0.0255 \\
\hline $\mathrm{C} 3 \mathrm{H}$ & $0.4607(3)$ & $0.7504(3)$ & $0.23808(13)$ & $0.0328(6)$ \\
\hline H3H1 & 0.4842 & 0.8161 & 0.2132 & $0.049 *$ \\
\hline $\mathrm{H} 3 \mathrm{H} 2$ & 0.5352 & 0.7320 & 0.2641 & $0.049^{*}$ \\
\hline Н3H3 & 0.3735 & 0.7801 & 0.2568 & $0.049^{*}$ \\
\hline O1I & 0.40709 (19) & $0.43145(18)$ & $0.27599(8)$ & 0.0280 \\
\hline H1I & 0.4267 & 0.4943 & 0.2583 & $0.042 *$ \\
\hline C1I & $0.5361(3)$ & $0.3481(3)$ & $0.27592(12)$ & $0.0294(6)$ \\
\hline H1I1 & 0.5877 & 0.3600 & 0.2423 & $0.035^{*}$ \\
\hline H1I2 & 0.5162 & 0.2591 & 0.2773 & $0.035^{*}$ \\
\hline $\mathrm{C} 2 \mathrm{I}$ & $0.6245(3)$ & $0.3723(3)$ & $0.32224(12)$ & $0.0327(6)$ \\
\hline $\mathrm{H} 2 \mathrm{I} 1$ & 0.5721 & 0.3626 & 0.3558 & $0.039^{*}$ \\
\hline $\mathrm{H} 2 \mathrm{I} 2$ & 0.7083 & 0.3088 & 0.3224 & $0.039^{*}$ \\
\hline $\mathrm{O} 2 \mathrm{I}$ & $0.6661(2)$ & $0.4974(2)$ & $0.31999(8)$ & $0.0334(5)$ \\
\hline C3I & $0.5953(4)$ & $0.5833(4)$ & $0.35674(13)$ & $0.0435(8)$ \\
\hline H3I1 & 0.4962 & 0.5968 & 0.3482 & $0.065^{*}$ \\
\hline H3I2 & 0.6334 & 0.6642 & 0.3546 & $0.065^{*}$ \\
\hline H3I3 & 0.6070 & 0.5485 & 0.3929 & $0.065^{*}$ \\
\hline O1J & 0.1679 (2) & $0.62932(17)$ & $0.28717(7)$ & $0.0235(4)$ \\
\hline H1J & 0.1135 & 0.6889 & 0.2740 & $0.035^{*}$ \\
\hline $\mathrm{C} 1 \mathrm{~J}$ & $0.1615(3)$ & $0.6484(3)$ & $0.34373(11)$ & $0.0279(6)$ \\
\hline H1J1 & 0.1530 & 0.7403 & 0.3513 & $0.033^{*}$ \\
\hline $\mathrm{H} 1 \mathrm{~J} 2$ & 0.2487 & 0.6079 & 0.3599 & $0.033^{*}$ \\
\hline $\mathrm{C} 2 \mathrm{~J}$ & $0.0403(3)$ & 0.5938 & $0.36864(11)$ & $0.0273(6)$ \\
\hline $\mathrm{H} 2 \mathrm{~J} 1$ & 0.0414 & 0.6034 & 0.4079 & $0.033^{*}$ \\
\hline
\end{tabular}


supporting information

\begin{tabular}{lllll} 
& & & \\
H2J2 & -0.0479 & 0.6391 & 0.3552 & $0.033^{*}$ \\
O2J & $0.0511(2)$ & $0.46206(17)$ & $0.35502(7)$ & $0.0238(4)$ \\
C3J & $-0.0263(3)$ & $0.3905(3)$ & $0.38985(11)$ & $0.0300(6)$ \\
H3J1 & 0.0141 & 0.3877 & 0.4255 & $0.045^{*}$ \\
H3J2 & -0.0234 & 0.3041 & 0.3762 & $0.045^{*}$ \\
H3J3 & -0.1229 & 0.4304 & 0.3918 & $0.045^{*}$ \\
\hline
\end{tabular}

Atomic displacement parameters $\left(\AA^{2}\right)$

\begin{tabular}{|c|c|c|c|c|c|c|}
\hline & $U^{11}$ & $U^{22}$ & $U^{33}$ & $U^{12}$ & $U^{13}$ & $U^{23}$ \\
\hline $\mathrm{Ba}$ & $0.01883(8)$ & $0.01347(8)$ & $0.01522(8)$ & $-0.00166(5)$ & $-0.00076(5)$ & $-0.00073(5)$ \\
\hline $\mathrm{Zn}$ & $0.01664(14)$ & $0.01436(14)$ & $0.01128(12)$ & $-0.00221(10)$ & $-0.00157(10)$ & $-0.00034(9)$ \\
\hline Cl1 & $0.0179(3)$ & $0.0180(3)$ & $0.0328(3)$ & $-0.0018(2)$ & $-0.0001(2)$ & $-0.0005(2)$ \\
\hline $\mathrm{Cl} 2$ & $0.0215(3)$ & $0.0179(3)$ & $0.0242(3)$ & $-0.0053(2)$ & $-0.0049(2)$ & $-0.0010(2)$ \\
\hline $\mathrm{O} 1$ & $0.0276(9)$ & $0.0181(8)$ & $0.0157(8)$ & $-0.0051(7)$ & $-0.0059(7)$ & $0.0044(6)$ \\
\hline $\mathrm{O} 2$ & $0.0229(9)$ & $0.0175(8)$ & $0.0136(7)$ & $-0.0042(7)$ & $-0.0039(7)$ & $-0.0004(6)$ \\
\hline $\mathrm{C} 1$ & $0.0158(11)$ & $0.0151(11)$ & $0.0127(10)$ & $0.0005(8)$ & $0.0001(8)$ & $-0.0002(8)$ \\
\hline $\mathrm{C} 2$ & $0.0152(11)$ & $0.0121(10)$ & $0.0123(10)$ & $-0.0030(8)$ & $-0.0005(8)$ & $0.0006(8)$ \\
\hline $\mathrm{C} 1 \mathrm{~A}$ & $0.0126(10)$ & $0.0153(11)$ & $0.0144(10)$ & $-0.0010(8)$ & $-0.0017(8)$ & $-0.0021(8)$ \\
\hline $\mathrm{C} 2 \mathrm{~A}$ & $0.0262(13)$ & $0.0184(12)$ & $0.0150(10)$ & $-0.0057(10)$ & $-0.0037(9)$ & $0.0002(9)$ \\
\hline $\mathrm{C} 3 \mathrm{~A}$ & $0.0340(15)$ & $0.0139(12)$ & $0.0234(12)$ & $-0.0017(10)$ & $-0.0070(11)$ & $-0.0015(9)$ \\
\hline $\mathrm{C} 4 \mathrm{~A}$ & $0.0302(14)$ & $0.0203(13)$ & $0.0227(12)$ & $0.0013(10)$ & $-0.0057(11)$ & $-0.0065(10)$ \\
\hline C5A & $0.0237(13)$ & $0.0279(13)$ & $0.0149(11)$ & $-0.0028(10)$ & $-0.0008(9)$ & $-0.0042(9)$ \\
\hline C6A & $0.0220(12)$ & $0.0181(11)$ & $0.0143(10)$ & $-0.0039(9)$ & $0.0004(9)$ & $-0.0018(9)$ \\
\hline $\mathrm{C} 1 \mathrm{~B}$ & $0.0211(12)$ & $0.0128(10)$ & $0.0110(9)$ & $-0.0042(9)$ & $-0.0008(8)$ & $-0.0018(8)$ \\
\hline $\mathrm{C} 2 \mathrm{~B}$ & $0.0221(12)$ & $0.0152(11)$ & $0.0152(10)$ & $-0.0018(9)$ & $-0.0033(9)$ & $-0.0012(8)$ \\
\hline $\mathrm{C} 3 \mathrm{~B}$ & $0.0323(14)$ & $0.0147(11)$ & $0.0184(11)$ & $-0.0040(10)$ & $-0.0043(10)$ & $0.0012(9)$ \\
\hline $\mathrm{C} 4 \mathrm{~B}$ & $0.0373(15)$ & $0.0184(12)$ & $0.0168(11)$ & $-0.0131(11)$ & $0.0029(10)$ & $0.0005(9)$ \\
\hline $\mathrm{C} 5 \mathrm{~B}$ & $0.0230(12)$ & $0.0228(12)$ & $0.0183(11)$ & $-0.0097(10)$ & $0.0045(9)$ & $-0.0038(9)$ \\
\hline C6B & $0.0196(12)$ & $0.0166(11)$ & $0.0162(10)$ & $0.0000(9)$ & $0.0012(9)$ & $-0.0021(9)$ \\
\hline $\mathrm{C} 1 \mathrm{C}$ & $0.0163(11)$ & $0.0136(10)$ & $0.0132(10)$ & $-0.0013(8)$ & $0.0016(8)$ & $0.0017(8)$ \\
\hline $\mathrm{C} 2 \mathrm{C}$ & $0.0203(12)$ & $0.0227(12)$ & $0.0132(10)$ & $-0.0032(10)$ & $0.0005(9)$ & $-0.0014(9)$ \\
\hline $\mathrm{C} 3 \mathrm{C}$ & $0.0178(12)$ & $0.0271(13)$ & $0.0203(11)$ & $-0.0046(10)$ & $-0.0033(9)$ & $0.0023(10)$ \\
\hline $\mathrm{C} 4 \mathrm{C}$ & $0.0151(12)$ & $0.0284(14)$ & $0.0255(13)$ & $-0.0003(10)$ & $0.0047(10)$ & $0.0026(10)$ \\
\hline $\mathrm{C} 5 \mathrm{C}$ & $0.0244(13)$ & $0.0321(15)$ & $0.0200(12)$ & $-0.0027(11)$ & $0.0071(10)$ & $-0.0068(10)$ \\
\hline $\mathrm{C} 6 \mathrm{C}$ & $0.0188(12)$ & $0.0251(13)$ & $0.0176(11)$ & $-0.0045(10)$ & $0.0009(9)$ & $-0.0061(9)$ \\
\hline $\mathrm{O} 3$ & $0.0364(11)$ & $0.0157(9)$ & $0.0210(9)$ & $-0.0033(8)$ & $0.0081(8)$ & $-0.0044(7)$ \\
\hline $\mathrm{O} 4$ & $0.0247(9)$ & $0.0196(9)$ & $0.0141(8)$ & $-0.0032(7)$ & $0.0025(7)$ & $0.0011(6)$ \\
\hline $\mathrm{C} 3$ & $0.0184(11)$ & $0.0199(12)$ & $0.0123(10)$ & $-0.0040(9)$ & $0.0000(9)$ & $0.0000(8)$ \\
\hline $\mathrm{C} 4$ & $0.0180(11)$ & $0.0159(11)$ & $0.0154(10)$ & $-0.0028(9)$ & $-0.0002(9)$ & $0.0007(8)$ \\
\hline C1D & $0.0248(13)$ & $0.0197(12)$ & $0.0131(10)$ & $-0.0059(10)$ & $-0.0019(9)$ & $-0.0012(9)$ \\
\hline $\mathrm{C} 2 \mathrm{D}$ & $0.0304(14)$ & $0.0247(13)$ & $0.0199(12)$ & $-0.0092(11)$ & $0.0040(10)$ & $-0.0016(10)$ \\
\hline C3D & $0.0497(19)$ & $0.0309(15)$ & $0.0241(13)$ & $-0.0219(13)$ & $0.0011(13)$ & $0.0016(11)$ \\
\hline C4D & $0.061(2)$ & $0.0227(14)$ & $0.0263(14)$ & $-0.0173(14)$ & $-0.0104(14)$ & $0.0082(11)$ \\
\hline C5D & $0.0477(19)$ & $0.0240(14)$ & $0.0355(16)$ & $-0.0046(13)$ & $-0.0183(14)$ & $0.0061(12)$ \\
\hline C6D & $0.0279(14)$ & $0.0238(13)$ & $0.0243(12)$ & $-0.0033(11)$ & $-0.0052(11)$ & $0.0025(10)$ \\
\hline $\mathrm{C} 1 \mathrm{E}$ & $0.0178(11)$ & $0.0209(12)$ & $0.0139(10)$ & $-0.0038(9)$ & $0.0010(9)$ & $0.0019(9)$ \\
\hline $\mathrm{C} 2 \mathrm{E}$ & $0.0226(13)$ & $0.0230(12)$ & $0.0147(10)$ & $-0.0039(10)$ & $0.0004(9)$ & $0.0022(9)$ \\
\hline
\end{tabular}




\begin{tabular}{|c|c|c|c|c|c|c|}
\hline $\mathrm{C} 3 \mathrm{E}$ & $0.0239(13)$ & $0.0275(14)$ & $0.0221(12)$ & $0.0002(11)$ & $-0.0008(10)$ & $0.0024(10)$ \\
\hline $\mathrm{C} 4 \mathrm{E}$ & $0.0201(13)$ & $0.0392(16)$ & $0.0241(13)$ & $-0.0008(11)$ & $-0.0027(10)$ & $0.0097(11)$ \\
\hline $\mathrm{C} 5 \mathrm{E}$ & $0.0267(14)$ & $0.0383(16)$ & $0.0215(12)$ & $-0.0105(12)$ & $-0.0067(11)$ & $-0.0003(11)$ \\
\hline C6E & $0.0246(13)$ & $0.0297(14)$ & $0.0213(12)$ & $-0.0077(11)$ & $-0.0010(10)$ & $-0.0029(10)$ \\
\hline $\mathrm{C} 1 \mathrm{~F}$ & $0.0172(11)$ & $0.0178(11)$ & $0.0149(10)$ & $-0.0014(9)$ & $-0.0021(9)$ & $-0.0023(8)$ \\
\hline $\mathrm{C} 2 \mathrm{~F}$ & $0.0316(14)$ & $0.0273(14)$ & $0.0173(11)$ & $-0.0092(11)$ & $0.0010(10)$ & $-0.0006(10)$ \\
\hline $\mathrm{C} 3 \mathrm{~F}$ & $0.0324(15)$ & $0.0331(15)$ & $0.0179(12)$ & $-0.0053(12)$ & $-0.0009(11)$ & $-0.0021(10)$ \\
\hline $\mathrm{C} 4 \mathrm{~F}$ & $0.0280(14)$ & $0.0361(16)$ & $0.0270(13)$ & $-0.0120(12)$ & $-0.0045(11)$ & $-0.0082(11)$ \\
\hline $\mathrm{C} 5 \mathrm{~F}$ & 0.0407 (17) & $0.0426(18)$ & $0.0274(14)$ & $-0.0269(14)$ & $0.0036(13)$ & $-0.0037(12)$ \\
\hline $\mathrm{C} 6 \mathrm{~F}$ & $0.0339(15)$ & $0.0336(15)$ & $0.0189(12)$ & $-0.0143(12)$ & $0.0023(11)$ & $-0.0029(10)$ \\
\hline $\mathrm{O} 1 \mathrm{G}$ & $0.0219(9)$ & $0.0263(10)$ & $0.0283(10)$ & $-0.0015(7)$ & $-0.0003(8)$ & $0.0034(8)$ \\
\hline $\mathrm{C} 1 \mathrm{G}$ & $0.0227(13)$ & $0.0237(13)$ & $0.0357(15)$ & $0.0000(11)$ & $-0.0055(11)$ & $0.0048(11)$ \\
\hline $\mathrm{C} 2 \mathrm{G}$ & $0.0265(14)$ & $0.0283(14)$ & $0.0341(15)$ & $-0.0010(11)$ & $-0.0035(12)$ & $0.0108(11)$ \\
\hline $\mathrm{O} 2 \mathrm{G}$ & $0.0281(10)$ & $0.0294(10)$ & $0.0291(10)$ & $0.0013(8)$ & $-0.0033(8)$ & $0.0011(8)$ \\
\hline $\mathrm{C} 3 \mathrm{G}$ & $0.0416(18)$ & $0.0429(18)$ & $0.0327(16)$ & $-0.0052(14)$ & $-0.0105(14)$ & $-0.0042(13)$ \\
\hline $\mathrm{O} 1 \mathrm{H}$ & $0.0540(14)$ & $0.0334(11)$ & $0.0229(10)$ & $-0.0244(10)$ & $0.0042(9)$ & $-0.0042(8)$ \\
\hline $\mathrm{C} 1 \mathrm{H}$ & $0.056(2)$ & $0.049(2)$ & $0.0284(15)$ & $-0.0296(17)$ & $0.0075(14)$ & $-0.0014(13)$ \\
\hline $\mathrm{C} 2 \mathrm{H}$ & 0.0457 (19) & $0.0329(16)$ & $0.0339(16)$ & $-0.0152(14)$ & $-0.0050(14)$ & $0.0050(12)$ \\
\hline $\mathrm{O} 2 \mathrm{H}$ & $0.0266(10)$ & $0.0213(9)$ & $0.0286(10)$ & $-0.0032(7)$ & $-0.0051(8)$ & $-0.0014(7)$ \\
\hline $\mathrm{C} 3 \mathrm{H}$ & $0.0376(17)$ & $0.0208(14)$ & $0.0404(16)$ & $-0.0056(12)$ & $0.0005(13)$ & $-0.0063(12)$ \\
\hline O1I & $0.0222(10)$ & $0.0286(10)$ & $0.0338(10)$ & $-0.0052(8)$ & $-0.0057(8)$ & $0.0094(8)$ \\
\hline C1I & $0.0243(14)$ & $0.0274(14)$ & $0.0356(15)$ & $-0.0003(11)$ & $0.0037(11)$ & $-0.0007(11)$ \\
\hline $\mathrm{C} 2 \mathrm{I}$ & $0.0244(14)$ & $0.0381(17)$ & $0.0343(15)$ & $0.0004(12)$ & $-0.0005(12)$ & $0.0103(13)$ \\
\hline $\mathrm{O} 2 \mathrm{I}$ & $0.0245(10)$ & $0.0441(13)$ & $0.0321(11)$ & $-0.0066(9)$ & $0.0032(8)$ & $-0.0031(9)$ \\
\hline $\mathrm{C} 3 \mathrm{I}$ & $0.046(2)$ & $0.055(2)$ & $0.0287(15)$ & $-0.0026(16)$ & $-0.0039(14)$ & $-0.0069(14)$ \\
\hline O1J & $0.0294(10)$ & $0.0184(9)$ & $0.0221(9)$ & $0.0001(7)$ & $-0.0021(8)$ & $-0.0024(7)$ \\
\hline $\mathrm{C} 1 \mathrm{~J}$ & $0.0347(15)$ & $0.0243(14)$ & $0.0251(13)$ & $-0.0052(11)$ & $-0.0037(11)$ & $-0.0033(10)$ \\
\hline $\mathrm{C} 2 \mathrm{~J}$ & $0.0327(15)$ & $0.0253(14)$ & $0.0231(12)$ & $-0.0003(11)$ & $0.0020(11)$ & $-0.0073(10)$ \\
\hline $\mathrm{O} 2 \mathrm{~J}$ & $0.0286(10)$ & $0.0231(9)$ & $0.0194(8)$ & $-0.0025(8)$ & $0.0035(7)$ & $0.0005(7)$ \\
\hline $\mathrm{C} 3 \mathrm{~J}$ & $0.0266(14)$ & $0.0391(16)$ & $0.0253(13)$ & $-0.0084(12)$ & $0.0014(11)$ & $0.0032(11)$ \\
\hline
\end{tabular}

Geometric parameters $\left(\stackrel{A}{ }{ }^{o}\right)$

\begin{tabular}{llll}
\hline $\mathrm{Ba}-\mathrm{O} 3$ & $2.6925(19)$ & $\mathrm{C} 1 \mathrm{E}-\mathrm{C} 2 \mathrm{E}$ & $1.387(3)$ \\
$\mathrm{Ba}-\mathrm{O} 1$ & $2.7073(19)$ & $\mathrm{C} 1 \mathrm{E}-\mathrm{C} 6 \mathrm{E}$ & $1.405(3)$ \\
$\mathrm{Ba}-\mathrm{O} 1 \mathrm{~J}$ & $2.7572(19)$ & $\mathrm{C} 2 \mathrm{E}-\mathrm{C} 3 \mathrm{E}$ & $1.389(4)$ \\
$\mathrm{Ba}-\mathrm{O} 1 \mathrm{H}$ & $2.783(2)$ & $\mathrm{C} 2 \mathrm{E}-\mathrm{H} 2 \mathrm{E}$ & 0.9500 \\
$\mathrm{Ba}-\mathrm{O} 2 \mathrm{~J}$ & $2.7908(19)$ & $\mathrm{C} 3 \mathrm{E}-\mathrm{C} 4 \mathrm{E}$ & $1.381(4)$ \\
$\mathrm{Ba}-\mathrm{O} 1 \mathrm{G}$ & $2.799(2)$ & $\mathrm{C} 3 \mathrm{E}-\mathrm{H} 3 \mathrm{E}$ & 0.9500 \\
$\mathrm{Ba}-\mathrm{O} 1 \mathrm{I}$ & $2.810(2)$ & $\mathrm{C} 4 \mathrm{E}-\mathrm{C} 5 \mathrm{E}$ & $1.392(4)$ \\
$\mathrm{Ba}-\mathrm{O} 2 \mathrm{G}$ & $2.985(2)$ & $\mathrm{C} 4 \mathrm{E}-\mathrm{H} 4 \mathrm{E}$ & 0.9500 \\
$\mathrm{Ba}-\mathrm{C} 11$ & $3.1118(11)$ & $\mathrm{C} 5 \mathrm{E}-\mathrm{C} 6 \mathrm{E}$ & $1.385(4)$ \\
$\mathrm{Ba}-\mathrm{Zn}$ & $3.9335(11)$ & $\mathrm{C} 5 \mathrm{E}-\mathrm{H} 5 \mathrm{E}$ & 0.9500 \\
$\mathrm{Zn}-\mathrm{O} 2$ & $1.9682(17)$ & $\mathrm{C} 6 \mathrm{E}-\mathrm{H} 6 \mathrm{E}$ & 0.9500 \\
$\mathrm{Zn}-\mathrm{O} 4$ & $1.9683(18)$ & $\mathrm{C} 1 \mathrm{~F}-\mathrm{C} 6 \mathrm{~F}$ & $1.388(4)$ \\
$\mathrm{Zn}-\mathrm{C} 11$ & $\mathrm{C} 1 \mathrm{~F}-\mathrm{C} 2 \mathrm{~F}$ & $1.400(3)$ \\
$\mathrm{Zn}-\mathrm{Cl2}$ & $2.2595(10)$ & $\mathrm{C} 2 \mathrm{~F}-\mathrm{C} 3 \mathrm{~F}$ & $1.386(4)$ \\
$\mathrm{O} 1-\mathrm{C} 1$ & $2.2653(9)$ & $\mathrm{C} 2 \mathrm{~F}-\mathrm{H} 2 \mathrm{~F}$ & 0.9500
\end{tabular}




\begin{tabular}{|c|c|c|c|}
\hline $\mathrm{O} 2-\mathrm{C} 1$ & $1.275(3)$ & $\mathrm{C} 3 \mathrm{~F}-\mathrm{C} 4 \mathrm{~F}$ & $1.372(4)$ \\
\hline $\mathrm{C} 1-\mathrm{C} 2$ & $1.568(3)$ & $\mathrm{C} 3 \mathrm{~F}-\mathrm{H} 3 \mathrm{~F}$ & 0.9500 \\
\hline $\mathrm{C} 2-\mathrm{C} 1 \mathrm{~B}$ & $1.531(3)$ & $\mathrm{C} 4 \mathrm{~F}-\mathrm{C} 5 \mathrm{~F}$ & $1.381(4)$ \\
\hline $\mathrm{C} 2-\mathrm{C} 1 \mathrm{~A}$ & $1.547(3)$ & $\mathrm{C} 4 \mathrm{~F}-\mathrm{H} 4 \mathrm{~F}$ & 0.9500 \\
\hline $\mathrm{C} 2-\mathrm{C} 1 \mathrm{C}$ & $1.550(3)$ & $\mathrm{C} 5 \mathrm{~F}-\mathrm{C} 6 \mathrm{~F}$ & $1.388(4)$ \\
\hline $\mathrm{C} 1 \mathrm{~A}-\mathrm{C} 6 \mathrm{~A}$ & $1.396(3)$ & $\mathrm{C} 5 \mathrm{~F}-\mathrm{H} 5 \mathrm{~F}$ & 0.9500 \\
\hline $\mathrm{C} 1 \mathrm{~A}-\mathrm{C} 2 \mathrm{~A}$ & $1.401(3)$ & $\mathrm{C} 6 \mathrm{~F}-\mathrm{H} 6 \mathrm{~F}$ & 0.9500 \\
\hline $\mathrm{C} 2 \mathrm{~A}-\mathrm{C} 3 \mathrm{~A}$ & $1.385(3)$ & $\mathrm{O} 1 \mathrm{G}-\mathrm{C} 1 \mathrm{G}$ & $1.434(3)$ \\
\hline $\mathrm{C} 2 \mathrm{~A}-\mathrm{H} 2 \mathrm{~A}$ & 0.9500 & $\mathrm{O} 1 \mathrm{G}-\mathrm{H} 1 \mathrm{G}$ & 0.8397 \\
\hline $\mathrm{C} 3 \mathrm{~A}-\mathrm{C} 4 \mathrm{~A}$ & $1.393(4)$ & $\mathrm{C} 1 \mathrm{G}-\mathrm{C} 2 \mathrm{G}$ & $1.500(4)$ \\
\hline $\mathrm{C} 3 \mathrm{~A}-\mathrm{H} 3 \mathrm{~A}$ & 0.9500 & $\mathrm{C} 1 \mathrm{G}-\mathrm{H} 1 \mathrm{G} 1$ & 0.9900 \\
\hline $\mathrm{C} 4 \mathrm{~A}-\mathrm{C} 5 \mathrm{~A}$ & $1.380(4)$ & $\mathrm{C} 1 \mathrm{G}-\mathrm{H} 1 \mathrm{G} 2$ & 0.9900 \\
\hline $\mathrm{C} 4 \mathrm{~A}-\mathrm{H} 4 \mathrm{~A}$ & 0.9500 & $\mathrm{C} 2 \mathrm{G}-\mathrm{O} 2 \mathrm{G}$ & $1.431(3)$ \\
\hline $\mathrm{C} 5 \mathrm{~A}-\mathrm{C} 6 \mathrm{~A}$ & $1.390(3)$ & $\mathrm{C} 2 \mathrm{G}-\mathrm{H} 2 \mathrm{G} 1$ & 0.9900 \\
\hline $\mathrm{C} 5 \mathrm{~A}-\mathrm{H} 5 \mathrm{~A}$ & 0.9500 & $\mathrm{C} 2 \mathrm{G}-\mathrm{H} 2 \mathrm{G} 2$ & 0.9900 \\
\hline $\mathrm{C} 6 \mathrm{~A}-\mathrm{H} 6 \mathrm{~A}$ & 0.9500 & $\mathrm{O} 2 \mathrm{G}-\mathrm{C} 3 \mathrm{G}$ & $1.422(4)$ \\
\hline $\mathrm{C} 1 \mathrm{~B}-\mathrm{C} 2 \mathrm{~B}$ & $1.391(3)$ & $\mathrm{C} 3 \mathrm{G}-\mathrm{H} 3 \mathrm{G} 1$ & 0.9800 \\
\hline $\mathrm{C} 1 \mathrm{~B}-\mathrm{C} 6 \mathrm{~B}$ & $1.397(3)$ & $\mathrm{C} 3 \mathrm{G}-\mathrm{H} 3 \mathrm{G} 2$ & 0.9800 \\
\hline $\mathrm{C} 2 \mathrm{~B}-\mathrm{C} 3 \mathrm{~B}$ & $1.394(3)$ & $\mathrm{C} 3 \mathrm{G}-\mathrm{H} 3 \mathrm{G} 3$ & 0.9800 \\
\hline $\mathrm{C} 2 \mathrm{~B}-\mathrm{H} 2 \mathrm{~B}$ & 0.9500 & $\mathrm{O} 1 \mathrm{H}-\mathrm{C} 1 \mathrm{H}$ & $1.407(4)$ \\
\hline $\mathrm{C} 3 \mathrm{~B}-\mathrm{C} 4 \mathrm{~B}$ & $1.381(4)$ & $\mathrm{O} 1 \mathrm{H}-\mathrm{H} 1 \mathrm{H}$ & 0.8402 \\
\hline $\mathrm{C} 3 \mathrm{~B}-\mathrm{H} 3 \mathrm{~B}$ & 0.9500 & $\mathrm{C} 1 \mathrm{H}-\mathrm{C} 2 \mathrm{H}$ & $1.487(4)$ \\
\hline $\mathrm{C} 4 \mathrm{~B}-\mathrm{C} 5 \mathrm{~B}$ & $1.389(4)$ & $\mathrm{C} 1 \mathrm{H}-\mathrm{H} 1 \mathrm{H} 1$ & 0.9900 \\
\hline $\mathrm{C} 4 \mathrm{~B}-\mathrm{H} 4 \mathrm{~B}$ & 0.9500 & $\mathrm{C} 1 \mathrm{H}-\mathrm{H} 1 \mathrm{H} 2$ & 0.9900 \\
\hline $\mathrm{C} 5 \mathrm{~B}-\mathrm{C} 6 \mathrm{~B}$ & $1.388(3)$ & $\mathrm{C} 2 \mathrm{H}-\mathrm{O} 2 \mathrm{H}$ & $1.440(4)$ \\
\hline $\mathrm{C} 5 \mathrm{~B}-\mathrm{H} 5 \mathrm{~B}$ & 0.9500 & $\mathrm{C} 2 \mathrm{H}-\mathrm{H} 2 \mathrm{H} 1$ & 0.9900 \\
\hline $\mathrm{C} 6 \mathrm{~B}-\mathrm{H} 6 \mathrm{~B}$ & 0.9500 & $\mathrm{C} 2 \mathrm{H}-\mathrm{H} 2 \mathrm{H} 2$ & 0.9900 \\
\hline $\mathrm{C} 1 \mathrm{C}-\mathrm{C} 2 \mathrm{C}$ & $1.390(3)$ & $\mathrm{O} 2 \mathrm{H}-\mathrm{C} 3 \mathrm{H}$ & $1.419(3)$ \\
\hline $\mathrm{C} 1 \mathrm{C}-\mathrm{C} 6 \mathrm{C}$ & $1.396(3)$ & $\mathrm{C} 3 \mathrm{H}-\mathrm{H} 3 \mathrm{H} 1$ & 0.9800 \\
\hline $\mathrm{C} 2 \mathrm{C}-\mathrm{C} 3 \mathrm{C}$ & $1.386(3)$ & $\mathrm{C} 3 \mathrm{H}-\mathrm{H} 3 \mathrm{H} 2$ & 0.9800 \\
\hline $\mathrm{C} 2 \mathrm{C}-\mathrm{H} 2 \mathrm{C}$ & 0.9500 & $\mathrm{C} 3 \mathrm{H}-\mathrm{H} 3 \mathrm{H} 3$ & 0.9800 \\
\hline $\mathrm{C} 3 \mathrm{C}-\mathrm{C} 4 \mathrm{C}$ & $1.390(4)$ & $\mathrm{O} 1 \mathrm{I}-\mathrm{C} 1 \mathrm{I}$ & $1.440(3)$ \\
\hline $\mathrm{C} 3 \mathrm{C}-\mathrm{H} 3 \mathrm{C}$ & 0.9500 & $\mathrm{O} 1 \mathrm{I}-\mathrm{H} 1 \mathrm{I}$ & 0.8399 \\
\hline $\mathrm{C} 4 \mathrm{C}-\mathrm{C} 5 \mathrm{C}$ & $1.387(4)$ & $\mathrm{C} 1 \mathrm{I}-\mathrm{C} 2 \mathrm{I}$ & $1.496(4)$ \\
\hline $\mathrm{C} 4 \mathrm{C}-\mathrm{H} 4 \mathrm{C}$ & 0.9500 & $\mathrm{C} 1 \mathrm{I}-\mathrm{H} 1 \mathrm{I} 1$ & 0.9900 \\
\hline $\mathrm{C} 5 \mathrm{C}-\mathrm{C} 6 \mathrm{C}$ & $1.388(4)$ & $\mathrm{C} 1 \mathrm{I}-\mathrm{H} 1 \mathrm{I} 2$ & 0.9900 \\
\hline $\mathrm{C} 5 \mathrm{C}-\mathrm{H} 5 \mathrm{C}$ & 0.9500 & $\mathrm{C} 2 \mathrm{I}-\mathrm{O} 2 \mathrm{I}$ & $1.439(4)$ \\
\hline $\mathrm{C} 6 \mathrm{C}-\mathrm{H} 6 \mathrm{C}$ & 0.9500 & $\mathrm{C} 2 \mathrm{I}-\mathrm{H} 2 \mathrm{I} 1$ & 0.9900 \\
\hline $\mathrm{O} 3-\mathrm{C} 3$ & $1.232(3)$ & $\mathrm{C} 2 \mathrm{I}-\mathrm{H} 2 \mathrm{I} 2$ & 0.9900 \\
\hline $\mathrm{O} 4-\mathrm{C} 3$ & $1.266(3)$ & $\mathrm{O} 2 \mathrm{I}-\mathrm{C} 3 \mathrm{I}$ & $1.412(4)$ \\
\hline $\mathrm{C} 3-\mathrm{C} 4$ & $1.565(3)$ & $\mathrm{C} 3 \mathrm{I}-\mathrm{H} 3 \mathrm{I} 1$ & 0.9800 \\
\hline $\mathrm{C} 4-\mathrm{C} 1 \mathrm{E}$ & $1.536(3)$ & $\mathrm{C} 3 \mathrm{I}-\mathrm{H} 3 \mathrm{I} 2$ & 0.9800 \\
\hline $\mathrm{C} 4-\mathrm{C} 1 \mathrm{D}$ & $1.540(3)$ & $\mathrm{C} 3 \mathrm{I}-\mathrm{H} 3 \mathrm{I} 3$ & 0.9800 \\
\hline $\mathrm{C} 4-\mathrm{C} 1 \mathrm{~F}$ & $1.549(3)$ & $\mathrm{O} 1 \mathrm{~J}-\mathrm{C} 1 \mathrm{~J}$ & $1.433(3)$ \\
\hline $\mathrm{C} 1 \mathrm{D}-\mathrm{C} 2 \mathrm{D}$ & $1.388(4)$ & $\mathrm{O} 1 \mathrm{~J}-\mathrm{H} 1 \mathrm{~J}$ & 0.8403 \\
\hline $\mathrm{C} 1 \mathrm{D}-\mathrm{C} 6 \mathrm{D}$ & $1.391(4)$ & $\mathrm{C} 1 \mathrm{~J}-\mathrm{C} 2 \mathrm{~J}$ & $1.503(4)$ \\
\hline $\mathrm{C} 2 \mathrm{D}-\mathrm{C} 3 \mathrm{D}$ & $1.392(4)$ & $\mathrm{C} 1 \mathrm{~J}-\mathrm{H} 1 \mathrm{~J} 1$ & 0.9900 \\
\hline $\mathrm{C} 2 \mathrm{D}-\mathrm{H} 2 \mathrm{D}$ & 0.9500 & $\mathrm{C} 1 \mathrm{~J}-\mathrm{H} 1 \mathrm{~J} 2$ & 0.9900 \\
\hline
\end{tabular}




\begin{tabular}{|c|c|c|c|}
\hline $\mathrm{C} 3 \mathrm{D}-\mathrm{C} 4 \mathrm{D}$ & $1.380(5)$ & $\mathrm{C} 2 \mathrm{~J}-\mathrm{O} 2 \mathrm{~J}$ & $1.436(3)$ \\
\hline $\mathrm{C} 3 \mathrm{D}-\mathrm{H} 3 \mathrm{D}$ & 0.9500 & $\mathrm{C} 2 \mathrm{~J}-\mathrm{H} 2 \mathrm{~J} 1$ & 0.9900 \\
\hline $\mathrm{C} 4 \mathrm{D}-\mathrm{C} 5 \mathrm{D}$ & $1.383(5)$ & $\mathrm{C} 2 \mathrm{~J}-\mathrm{H} 2 \mathrm{~J} 2$ & 0.9900 \\
\hline $\mathrm{C} 4 \mathrm{D}-\mathrm{H} 4 \mathrm{D}$ & 0.9500 & $\mathrm{O} 2 \mathrm{~J}-\mathrm{C} 3 \mathrm{~J}$ & $1.424(3)$ \\
\hline $\mathrm{C} 5 \mathrm{D}-\mathrm{C} 6 \mathrm{D}$ & $1.390(4)$ & $\mathrm{C} 3 \mathrm{~J}-\mathrm{H} 3 \mathrm{~J} 1$ & 0.9800 \\
\hline $\mathrm{C} 5 \mathrm{D}-\mathrm{H} 5 \mathrm{D}$ & 0.9500 & $\mathrm{C} 3 \mathrm{~J}-\mathrm{H} 3 \mathrm{~J} 2$ & 0.9800 \\
\hline C6D-H6D & 0.9500 & $\mathrm{C} 3 \mathrm{~J}-\mathrm{H} 3 \mathrm{~J} 3$ & 0.9800 \\
\hline $\mathrm{O} 3-\mathrm{Ba}-\mathrm{O} 1$ & $84.08(6)$ & $\mathrm{C} 4 \mathrm{D}-\mathrm{C} 3 \mathrm{D}-\mathrm{H} 3 \mathrm{D}$ & 119.5 \\
\hline $\mathrm{O} 3-\mathrm{Ba}-\mathrm{O} 1 \mathrm{~J}$ & $142.04(6)$ & $\mathrm{C} 2 \mathrm{D}-\mathrm{C} 3 \mathrm{D}-\mathrm{H} 3 \mathrm{D}$ & 119.5 \\
\hline $\mathrm{O} 1-\mathrm{Ba}-\mathrm{O} 1 \mathrm{~J}$ & $114.85(6)$ & $\mathrm{C} 3 \mathrm{D}-\mathrm{C} 4 \mathrm{D}-\mathrm{C} 5 \mathrm{D}$ & $118.6(3)$ \\
\hline $\mathrm{O} 3-\mathrm{Ba}-\mathrm{O} 1 \mathrm{H}$ & $60.16(6)$ & $\mathrm{C} 3 \mathrm{D}-\mathrm{C} 4 \mathrm{D}-\mathrm{H} 4 \mathrm{D}$ & 120.7 \\
\hline $\mathrm{O} 1-\mathrm{Ba}-\mathrm{O} 1 \mathrm{H}$ & $122.95(7)$ & $\mathrm{C} 5 \mathrm{D}-\mathrm{C} 4 \mathrm{D}-\mathrm{H} 4 \mathrm{D}$ & 120.7 \\
\hline $\mathrm{O} 1 \mathrm{~J}-\mathrm{Ba}-\mathrm{O} 1 \mathrm{H}$ & $82.35(6)$ & $\mathrm{C} 4 \mathrm{D}-\mathrm{C} 5 \mathrm{D}-\mathrm{C} 6 \mathrm{D}$ & $120.7(3)$ \\
\hline $\mathrm{O} 3-\mathrm{Ba}-\mathrm{O} 2 \mathrm{~J}$ & $155.84(6)$ & $\mathrm{C} 4 \mathrm{D}-\mathrm{C} 5 \mathrm{D}-\mathrm{H} 5 \mathrm{D}$ & 119.7 \\
\hline $\mathrm{O} 1-\mathrm{Ba}-\mathrm{O} 2 \mathrm{~J}$ & $74.87(6)$ & $\mathrm{C} 6 \mathrm{D}-\mathrm{C} 5 \mathrm{D}-\mathrm{H} 5 \mathrm{D}$ & 119.7 \\
\hline $\mathrm{O} 1 \mathrm{~J}-\mathrm{Ba}-\mathrm{O} 2 \mathrm{~J}$ & $60.31(6)$ & $\mathrm{C} 5 \mathrm{D}-\mathrm{C} 6 \mathrm{D}-\mathrm{C} 1 \mathrm{D}$ & $121.2(3)$ \\
\hline $\mathrm{O} 1 \mathrm{H}-\mathrm{Ba}-\mathrm{O} 2 \mathrm{~J}$ & $142.49(6)$ & $\mathrm{C} 5 \mathrm{D}-\mathrm{C} 6 \mathrm{D}-\mathrm{H} 6 \mathrm{D}$ & 119.4 \\
\hline $\mathrm{O} 3-\mathrm{Ba}-\mathrm{O} 1 \mathrm{G}$ & $120.96(6)$ & $\mathrm{C} 1 \mathrm{D}-\mathrm{C} 6 \mathrm{D}-\mathrm{H} 6 \mathrm{D}$ & 119.4 \\
\hline $\mathrm{O} 1-\mathrm{Ba}-\mathrm{O} 1 \mathrm{G}$ & $133.04(6)$ & $\mathrm{C} 2 \mathrm{E}-\mathrm{C} 1 \mathrm{E}-\mathrm{C} 6 \mathrm{E}$ & $118.1(2)$ \\
\hline $\mathrm{O} 1 \mathrm{~J}-\mathrm{Ba}-\mathrm{O} 1 \mathrm{G}$ & $71.05(6)$ & $\mathrm{C} 2 \mathrm{E}-\mathrm{C} 1 \mathrm{E}-\mathrm{C} 4$ & $122.6(2)$ \\
\hline $\mathrm{O} 1 \mathrm{H}-\mathrm{Ba}-\mathrm{O} 1 \mathrm{G}$ & $103.93(7)$ & $\mathrm{C} 6 \mathrm{E}-\mathrm{C} 1 \mathrm{E}-\mathrm{C} 4$ & $119.3(2)$ \\
\hline $\mathrm{O} 2 \mathrm{~J}-\mathrm{Ba}-\mathrm{O} 1 \mathrm{G}$ & $68.94(6)$ & $\mathrm{C} 1 \mathrm{E}-\mathrm{C} 2 \mathrm{E}-\mathrm{C} 3 \mathrm{E}$ & $120.8(2)$ \\
\hline $\mathrm{O} 3-\mathrm{Ba}-\mathrm{O} 1 \mathrm{I}$ & $95.18(7)$ & $\mathrm{C} 1 \mathrm{E}-\mathrm{C} 2 \mathrm{E}-\mathrm{H} 2 \mathrm{E}$ & 119.6 \\
\hline $\mathrm{O} 1-\mathrm{Ba}-\mathrm{O} 1 \mathrm{I}$ & $71.58(6)$ & $\mathrm{C} 3 \mathrm{E}-\mathrm{C} 2 \mathrm{E}-\mathrm{H} 2 \mathrm{E}$ & 119.6 \\
\hline $\mathrm{O} 1 \mathrm{~J}-\mathrm{Ba}-\mathrm{O} 1 \mathrm{I}$ & $63.80(6)$ & $\mathrm{C} 4 \mathrm{E}-\mathrm{C} 3 \mathrm{E}-\mathrm{C} 2 \mathrm{E}$ & $120.7(3)$ \\
\hline $\mathrm{O} 1 \mathrm{H}-\mathrm{Ba}-\mathrm{O} 1 \mathrm{I}$ & $69.50(7)$ & $\mathrm{C} 4 \mathrm{E}-\mathrm{C} 3 \mathrm{E}-\mathrm{H} 3 \mathrm{E}$ & 119.7 \\
\hline $\mathrm{O} 2 \mathrm{~J}-\mathrm{Ba}-\mathrm{O} 1 \mathrm{I}$ & $89.38(6)$ & $\mathrm{C} 2 \mathrm{E}-\mathrm{C} 3 \mathrm{E}-\mathrm{H} 3 \mathrm{E}$ & 119.7 \\
\hline $\mathrm{O} 1 \mathrm{G}-\mathrm{Ba}-\mathrm{O} 1 \mathrm{I}$ & $134.83(6)$ & $\mathrm{C} 3 \mathrm{E}-\mathrm{C} 4 \mathrm{E}-\mathrm{C} 5 \mathrm{E}$ & $119.6(3)$ \\
\hline $\mathrm{O} 3-\mathrm{Ba}-\mathrm{O} 2 \mathrm{G}$ & $65.59(6)$ & $\mathrm{C} 3 \mathrm{E}-\mathrm{C} 4 \mathrm{E}-\mathrm{H} 4 \mathrm{E}$ & 120.2 \\
\hline $\mathrm{O} 1-\mathrm{Ba}-\mathrm{O} 2 \mathrm{G}$ & $139.45(6)$ & $\mathrm{C} 5 \mathrm{E}-\mathrm{C} 4 \mathrm{E}-\mathrm{H} 4 \mathrm{E}$ & 120.2 \\
\hline $\mathrm{O} 1 \mathrm{~J}-\mathrm{Ba}-\mathrm{O} 2 \mathrm{G}$ & $105.51(6)$ & $\mathrm{C} 6 \mathrm{E}-\mathrm{C} 5 \mathrm{E}-\mathrm{C} 4 \mathrm{E}$ & $119.7(3)$ \\
\hline $\mathrm{O} 1 \mathrm{H}-\mathrm{Ba}-\mathrm{O} 2 \mathrm{G}$ & $64.98(6)$ & $\mathrm{C} 6 \mathrm{E}-\mathrm{C} 5 \mathrm{E}-\mathrm{H} 5 \mathrm{E}$ & 120.2 \\
\hline $\mathrm{O} 2 \mathrm{~J}-\mathrm{Ba}-\mathrm{O} 2 \mathrm{G}$ & $125.48(6)$ & $\mathrm{C} 4 \mathrm{E}-\mathrm{C} 5 \mathrm{E}-\mathrm{H} 5 \mathrm{E}$ & 120.2 \\
\hline $\mathrm{O} 1 \mathrm{G}-\mathrm{Ba}-\mathrm{O} 2 \mathrm{G}$ & $57.15(6)$ & $\mathrm{C} 5 \mathrm{E}-\mathrm{C} 6 \mathrm{E}-\mathrm{C} 1 \mathrm{E}$ & $121.2(3)$ \\
\hline $\mathrm{O} 1 \mathrm{I}-\mathrm{Ba}-\mathrm{O} 2 \mathrm{G}$ & $134.31(6)$ & $\mathrm{C} 5 \mathrm{E}-\mathrm{C} 6 \mathrm{E}-\mathrm{H} 6 \mathrm{E}$ & 119.4 \\
\hline $\mathrm{O} 3-\mathrm{Ba}-\mathrm{Cl1}$ & $74.73(5)$ & $\mathrm{C} 1 \mathrm{E}-\mathrm{C} 6 \mathrm{E}-\mathrm{H} 6 \mathrm{E}$ & 119.4 \\
\hline $\mathrm{O} 1-\mathrm{Ba}-\mathrm{Cl1}$ & $71.96(5)$ & $\mathrm{C} 6 \mathrm{~F}-\mathrm{C} 1 \mathrm{~F}-\mathrm{C} 2 \mathrm{~F}$ & $117.0(2)$ \\
\hline $\mathrm{O} 1 \mathrm{~J}-\mathrm{Ba}-\mathrm{Cl} 1$ & $140.95(4)$ & $\mathrm{C} 6 \mathrm{~F}-\mathrm{C} 1 \mathrm{~F}-\mathrm{C} 4$ & $124.4(2)$ \\
\hline $\mathrm{O} 1 \mathrm{H}-\mathrm{Ba}-\mathrm{Cl} 1$ & $128.16(5)$ & $\mathrm{C} 2 \mathrm{~F}-\mathrm{C} 1 \mathrm{~F}-\mathrm{C} 4$ & $118.6(2)$ \\
\hline $\mathrm{O} 2 \mathrm{~J}-\mathrm{Ba}-\mathrm{Cl} 1$ & $87.39(5)$ & $\mathrm{C} 3 \mathrm{~F}-\mathrm{C} 2 \mathrm{~F}-\mathrm{C} 1 \mathrm{~F}$ & $121.7(2)$ \\
\hline $\mathrm{O} 1 \mathrm{G}-\mathrm{Ba}-\mathrm{Cl} 1$ & $77.39(5)$ & $\mathrm{C} 3 \mathrm{~F}-\mathrm{C} 2 \mathrm{~F}-\mathrm{H} 2 \mathrm{~F}$ & 119.2 \\
\hline $\mathrm{O} 1 \mathrm{I}-\mathrm{Ba}-\mathrm{Cl1}$ & $142.95(4)$ & $\mathrm{C} 1 \mathrm{~F}-\mathrm{C} 2 \mathrm{~F}-\mathrm{H} 2 \mathrm{~F}$ & 119.2 \\
\hline $\mathrm{O} 2 \mathrm{G}-\mathrm{Ba}-\mathrm{Cl} 1$ & $74.38(5)$ & $\mathrm{C} 4 \mathrm{~F}-\mathrm{C} 3 \mathrm{~F}-\mathrm{C} 2 \mathrm{~F}$ & $120.7(2)$ \\
\hline $\mathrm{O} 3-\mathrm{Ba}-\mathrm{Zn}$ & $53.90(4)$ & $\mathrm{C} 4 \mathrm{~F}-\mathrm{C} 3 \mathrm{~F}-\mathrm{H} 3 \mathrm{~F}$ & 119.6 \\
\hline $\mathrm{O} 1-\mathrm{Ba}-\mathrm{Zn}$ & $48.34(4)$ & $\mathrm{C} 2 \mathrm{~F}-\mathrm{C} 3 \mathrm{~F}-\mathrm{H} 3 \mathrm{~F}$ & 119.6 \\
\hline $\mathrm{O} 1 \mathrm{~J}-\mathrm{Ba}-\mathrm{Zn}$ & $160.81(4)$ & $\mathrm{C} 3 \mathrm{~F}-\mathrm{C} 4 \mathrm{~F}-\mathrm{C} 5 \mathrm{~F}$ & $118.2(3)$ \\
\hline $\mathrm{O} 1 \mathrm{H}-\mathrm{Ba}-\mathrm{Zn}$ & $113.88(5)$ & $\mathrm{C} 3 \mathrm{~F}-\mathrm{C} 4 \mathrm{~F}-\mathrm{H} 4 \mathrm{~F}$ & 120.9 \\
\hline
\end{tabular}




\begin{tabular}{|c|c|c|c|}
\hline $\mathrm{O} 2 \mathrm{~J}-\mathrm{Ba}-\mathrm{Zn}$ & $102.37(5)$ & $\mathrm{C} 5 \mathrm{~F}-\mathrm{C} 4 \mathrm{~F}-\mathrm{H} 4 \mathrm{~F}$ & 120.9 \\
\hline $\mathrm{O} 1 \mathrm{G}-\mathrm{Ba}-\mathrm{Zn}$ & $112.26(5)$ & $\mathrm{C} 4 \mathrm{~F}-\mathrm{C} 5 \mathrm{~F}-\mathrm{C} 6 \mathrm{~F}$ & $121.6(3)$ \\
\hline $\mathrm{O} 1 \mathrm{I}-\mathrm{Ba}-\mathrm{Zn}$ & $110.85(5)$ & $\mathrm{C} 4 \mathrm{~F}-\mathrm{C} 5 \mathrm{~F}-\mathrm{H} 5 \mathrm{~F}$ & 119.2 \\
\hline $\mathrm{O} 2 \mathrm{G}-\mathrm{Ba}-\mathrm{Zn}$ & $91.21(5)$ & $\mathrm{C} 6 \mathrm{~F}-\mathrm{C} 5 \mathrm{~F}-\mathrm{H} 5 \mathrm{~F}$ & 119.2 \\
\hline $\mathrm{Cl} 1-\mathrm{Ba}-\mathrm{Zn}$ & $35.01(2)$ & $\mathrm{C} 1 \mathrm{~F}-\mathrm{C} 6 \mathrm{~F}-\mathrm{C} 5 \mathrm{~F}$ & $120.7(2)$ \\
\hline $\mathrm{O} 2-\mathrm{Zn}-\mathrm{O} 4$ & $110.00(8)$ & $\mathrm{C} 1 \mathrm{~F}-\mathrm{C} 6 \mathrm{~F}-\mathrm{H} 6 \mathrm{~F}$ & 119.6 \\
\hline $\mathrm{O} 2-\mathrm{Zn}-\mathrm{Cl1}$ & $111.30(6)$ & $\mathrm{C} 5 \mathrm{~F}-\mathrm{C} 6 \mathrm{~F}-\mathrm{H} 6 \mathrm{~F}$ & 119.6 \\
\hline $\mathrm{O} 4-\mathrm{Zn}-\mathrm{Cl1}$ & $118.74(6)$ & $\mathrm{C} 1 \mathrm{G}-\mathrm{O} 1 \mathrm{G}-\mathrm{Ba}$ & $124.47(16)$ \\
\hline $\mathrm{O} 2-\mathrm{Zn}-\mathrm{Cl} 2$ & $107.44(6)$ & $\mathrm{C} 1 \mathrm{G}-\mathrm{O} 1 \mathrm{G}-\mathrm{H} 1 \mathrm{G}$ & 111.8 \\
\hline $\mathrm{O} 4-\mathrm{Zn}-\mathrm{Cl} 2$ & $102.14(6)$ & $\mathrm{Ba}-\mathrm{O} 1 \mathrm{G}-\mathrm{H} 1 \mathrm{G}$ & 119.0 \\
\hline $\mathrm{Cl} 1-\mathrm{Zn}-\mathrm{Cl} 2$ & $106.19(3)$ & $\mathrm{O} 1 \mathrm{G}-\mathrm{C} 1 \mathrm{G}-\mathrm{C} 2 \mathrm{G}$ & $107.4(2)$ \\
\hline $\mathrm{O} 2-\mathrm{Zn}-\mathrm{Ba}$ & $91.95(6)$ & $\mathrm{O} 1 \mathrm{G}-\mathrm{C} 1 \mathrm{G}-\mathrm{H} 1 \mathrm{G} 1$ & 110.2 \\
\hline $\mathrm{O} 4-\mathrm{Zn}-\mathrm{Ba}$ & $83.59(6)$ & $\mathrm{C} 2 \mathrm{G}-\mathrm{C} 1 \mathrm{G}-\mathrm{H} 1 \mathrm{G} 1$ & 110.2 \\
\hline $\mathrm{C} 11-\mathrm{Zn}-\mathrm{Ba}$ & $52.20(3)$ & $\mathrm{O} 1 \mathrm{G}-\mathrm{C} 1 \mathrm{G}-\mathrm{H} 1 \mathrm{G} 2$ & 110.2 \\
\hline $\mathrm{Cl} 2-\mathrm{Zn}-\mathrm{Ba}$ & $156.02(2)$ & $\mathrm{C} 2 \mathrm{G}-\mathrm{C} 1 \mathrm{G}-\mathrm{H} 1 \mathrm{G} 2$ & 110.2 \\
\hline $\mathrm{Zn}-\mathrm{Cl1}-\mathrm{Ba}$ & $92.79(3)$ & $\mathrm{H} 1 \mathrm{G} 1-\mathrm{C} 1 \mathrm{G}-\mathrm{H} 1 \mathrm{G} 2$ & 108.5 \\
\hline $\mathrm{C} 1-\mathrm{O} 1-\mathrm{Ba}$ & $155.72(16)$ & $\mathrm{O} 2 \mathrm{G}-\mathrm{C} 2 \mathrm{G}-\mathrm{C} 1 \mathrm{G}$ & $113.0(2)$ \\
\hline $\mathrm{C} 1-\mathrm{O} 2-\mathrm{Zn}$ & $116.35(15)$ & $\mathrm{O} 2 \mathrm{G}-\mathrm{C} 2 \mathrm{G}-\mathrm{H} 2 \mathrm{G} 1$ & 109.0 \\
\hline $\mathrm{O} 1-\mathrm{C} 1-\mathrm{O} 2$ & $124.3(2)$ & $\mathrm{C} 1 \mathrm{G}-\mathrm{C} 2 \mathrm{G}-\mathrm{H} 2 \mathrm{G} 1$ & 109.0 \\
\hline $\mathrm{O} 1-\mathrm{C} 1-\mathrm{C} 2$ & $120.6(2)$ & $\mathrm{O} 2 \mathrm{G}-\mathrm{C} 2 \mathrm{G}-\mathrm{H} 2 \mathrm{G} 2$ & 109.0 \\
\hline $\mathrm{O} 2-\mathrm{C} 1-\mathrm{C} 2$ & $115.06(19)$ & $\mathrm{C} 1 \mathrm{G}-\mathrm{C} 2 \mathrm{G}-\mathrm{H} 2 \mathrm{G} 2$ & 109.0 \\
\hline $\mathrm{C} 1 \mathrm{~B}-\mathrm{C} 2-\mathrm{C} 1 \mathrm{~A}$ & $109.37(18)$ & $\mathrm{H} 2 \mathrm{G} 1-\mathrm{C} 2 \mathrm{G}-\mathrm{H} 2 \mathrm{G} 2$ & 107.8 \\
\hline $\mathrm{C} 1 \mathrm{~B}-\mathrm{C} 2-\mathrm{C} 1 \mathrm{C}$ & $110.82(18)$ & $\mathrm{C} 3 \mathrm{G}-\mathrm{O} 2 \mathrm{G}-\mathrm{C} 2 \mathrm{G}$ & $113.5(2)$ \\
\hline $\mathrm{C} 1 \mathrm{~A}-\mathrm{C} 2-\mathrm{C} 1 \mathrm{C}$ & $108.82(18)$ & $\mathrm{C} 3 \mathrm{G}-\mathrm{O} 2 \mathrm{G}-\mathrm{Ba}$ & $125.66(17)$ \\
\hline $\mathrm{C} 1 \mathrm{~B}-\mathrm{C} 2-\mathrm{C} 1$ & $109.40(18)$ & $\mathrm{C} 2 \mathrm{G}-\mathrm{O} 2 \mathrm{G}-\mathrm{Ba}$ & $102.89(15)$ \\
\hline $\mathrm{C} 1 \mathrm{~A}-\mathrm{C} 2-\mathrm{C} 1$ & $112.19(18)$ & $\mathrm{O} 2 \mathrm{G}-\mathrm{C} 3 \mathrm{G}-\mathrm{H} 3 \mathrm{G} 1$ & 109.5 \\
\hline $\mathrm{C} 1 \mathrm{C}-\mathrm{C} 2-\mathrm{C} 1$ & $106.20(17)$ & $\mathrm{O} 2 \mathrm{G}-\mathrm{C} 3 \mathrm{G}-\mathrm{H} 3 \mathrm{G} 2$ & 109.5 \\
\hline $\mathrm{C} 6 \mathrm{~A}-\mathrm{C} 1 \mathrm{~A}-\mathrm{C} 2 \mathrm{~A}$ & $117.2(2)$ & $\mathrm{H} 3 \mathrm{G} 1-\mathrm{C} 3 \mathrm{G}-\mathrm{H} 3 \mathrm{G} 2$ & 109.5 \\
\hline $\mathrm{C} 6 \mathrm{~A}-\mathrm{C} 1 \mathrm{~A}-\mathrm{C} 2$ & $121.6(2)$ & $\mathrm{O} 2 \mathrm{G}-\mathrm{C} 3 \mathrm{G}-\mathrm{H} 3 \mathrm{G} 3$ & 109.5 \\
\hline $\mathrm{C} 2 \mathrm{~A}-\mathrm{C} 1 \mathrm{~A}-\mathrm{C} 2$ & $121.0(2)$ & $\mathrm{H} 3 \mathrm{G} 1-\mathrm{C} 3 \mathrm{G}-\mathrm{H} 3 \mathrm{G} 3$ & 109.5 \\
\hline $\mathrm{C} 3 \mathrm{~A}-\mathrm{C} 2 \mathrm{~A}-\mathrm{C} 1 \mathrm{~A}$ & $120.8(2)$ & $\mathrm{H} 3 \mathrm{G} 2-\mathrm{C} 3 \mathrm{G}-\mathrm{H} 3 \mathrm{G} 3$ & 109.5 \\
\hline $\mathrm{C} 3 \mathrm{~A}-\mathrm{C} 2 \mathrm{~A}-\mathrm{H} 2 \mathrm{~A}$ & 119.6 & $\mathrm{C} 1 \mathrm{H}-\mathrm{O} 1 \mathrm{H}-\mathrm{Ba}$ & $152.75(18)$ \\
\hline $\mathrm{C} 1 \mathrm{~A}-\mathrm{C} 2 \mathrm{~A}-\mathrm{H} 2 \mathrm{~A}$ & 119.6 & $\mathrm{C} 1 \mathrm{H}-\mathrm{O} 1 \mathrm{H}-\mathrm{H} 1 \mathrm{H}$ & 108.3 \\
\hline $\mathrm{C} 2 \mathrm{~A}-\mathrm{C} 3 \mathrm{~A}-\mathrm{C} 4 \mathrm{~A}$ & $121.1(2)$ & $\mathrm{Ba}-\mathrm{O} 1 \mathrm{H}-\mathrm{H} 1 \mathrm{H}$ & 98.5 \\
\hline $\mathrm{C} 2 \mathrm{~A}-\mathrm{C} 3 \mathrm{~A}-\mathrm{H} 3 \mathrm{~A}$ & 119.5 & $\mathrm{O} 1 \mathrm{H}-\mathrm{C} 1 \mathrm{H}-\mathrm{C} 2 \mathrm{H}$ & $109.1(3)$ \\
\hline $\mathrm{C} 4 \mathrm{~A}-\mathrm{C} 3 \mathrm{~A}-\mathrm{H} 3 \mathrm{~A}$ & 119.5 & $\mathrm{O} 1 \mathrm{H}-\mathrm{C} 1 \mathrm{H}-\mathrm{H} 1 \mathrm{H} 1$ & 109.9 \\
\hline $\mathrm{C} 5 \mathrm{~A}-\mathrm{C} 4 \mathrm{~A}-\mathrm{C} 3 \mathrm{~A}$ & $118.8(2)$ & $\mathrm{C} 2 \mathrm{H}-\mathrm{C} 1 \mathrm{H}-\mathrm{H} 1 \mathrm{H} 1$ & 109.9 \\
\hline $\mathrm{C} 5 \mathrm{~A}-\mathrm{C} 4 \mathrm{~A}-\mathrm{H} 4 \mathrm{~A}$ & 120.6 & $\mathrm{O} 1 \mathrm{H}-\mathrm{C} 1 \mathrm{H}-\mathrm{H} 1 \mathrm{H} 2$ & 109.9 \\
\hline $\mathrm{C} 3 \mathrm{~A}-\mathrm{C} 4 \mathrm{~A}-\mathrm{H} 4 \mathrm{~A}$ & 120.6 & $\mathrm{C} 2 \mathrm{H}-\mathrm{C} 1 \mathrm{H}-\mathrm{H} 1 \mathrm{H} 2$ & 109.9 \\
\hline $\mathrm{C} 4 \mathrm{~A}-\mathrm{C} 5 \mathrm{~A}-\mathrm{C} 6 \mathrm{~A}$ & $120.2(2)$ & $\mathrm{H} 1 \mathrm{H} 1-\mathrm{C} 1 \mathrm{H}-\mathrm{H} 1 \mathrm{H} 2$ & 108.3 \\
\hline $\mathrm{C} 4 \mathrm{~A}-\mathrm{C} 5 \mathrm{~A}-\mathrm{H} 5 \mathrm{~A}$ & 119.9 & $\mathrm{O} 2 \mathrm{H}-\mathrm{C} 2 \mathrm{H}-\mathrm{C} 1 \mathrm{H}$ & $108.8(3)$ \\
\hline $\mathrm{C} 6 \mathrm{~A}-\mathrm{C} 5 \mathrm{~A}-\mathrm{H} 5 \mathrm{~A}$ & 119.9 & $\mathrm{O} 2 \mathrm{H}-\mathrm{C} 2 \mathrm{H}-\mathrm{H} 2 \mathrm{H} 1$ & 109.9 \\
\hline $\mathrm{C} 5 \mathrm{~A}-\mathrm{C} 6 \mathrm{~A}-\mathrm{C} 1 \mathrm{~A}$ & $121.9(2)$ & $\mathrm{C} 1 \mathrm{H}-\mathrm{C} 2 \mathrm{H}-\mathrm{H} 2 \mathrm{H} 1$ & 109.9 \\
\hline $\mathrm{C} 5 \mathrm{~A}-\mathrm{C} 6 \mathrm{~A}-\mathrm{H} 6 \mathrm{~A}$ & 119.0 & $\mathrm{O} 2 \mathrm{H}-\mathrm{C} 2 \mathrm{H}-\mathrm{H} 2 \mathrm{H} 2$ & 109.9 \\
\hline $\mathrm{C} 1 \mathrm{~A}-\mathrm{C} 6 \mathrm{~A}-\mathrm{H} 6 \mathrm{~A}$ & 119.0 & $\mathrm{C} 1 \mathrm{H}-\mathrm{C} 2 \mathrm{H}-\mathrm{H} 2 \mathrm{H} 2$ & 109.9 \\
\hline $\mathrm{C} 2 \mathrm{~B}-\mathrm{C} 1 \mathrm{~B}-\mathrm{C} 6 \mathrm{~B}$ & $117.7(2)$ & $\mathrm{H} 2 \mathrm{H} 1-\mathrm{C} 2 \mathrm{H}-\mathrm{H} 2 \mathrm{H} 2$ & 108.3 \\
\hline $\mathrm{C} 2 \mathrm{~B}-\mathrm{C} 1 \mathrm{~B}-\mathrm{C} 2$ & $123.1(2)$ & $\mathrm{C} 3 \mathrm{H}-\mathrm{O} 2 \mathrm{H}-\mathrm{C} 2 \mathrm{H}$ & $108.2(2)$ \\
\hline $\mathrm{C} 6 \mathrm{~B}-\mathrm{C} 1 \mathrm{~B}-\mathrm{C} 2$ & $119.1(2)$ & $\mathrm{O} 2 \mathrm{H}-\mathrm{C} 3 \mathrm{H}-\mathrm{H} 3 \mathrm{H} 1$ & 109.5 \\
\hline
\end{tabular}


$\mathrm{C} 1 \mathrm{~B}-\mathrm{C} 2 \mathrm{~B}-\mathrm{C} 3 \mathrm{~B}$

$\mathrm{C} 1 \mathrm{~B}-\mathrm{C} 2 \mathrm{~B}-\mathrm{H} 2 \mathrm{~B}$

$\mathrm{C} 3 \mathrm{~B}-\mathrm{C} 2 \mathrm{~B}-\mathrm{H} 2 \mathrm{~B}$

$\mathrm{C} 4 \mathrm{~B}-\mathrm{C} 3 \mathrm{~B}-\mathrm{C} 2 \mathrm{~B}$

$\mathrm{C} 4 \mathrm{~B}-\mathrm{C} 3 \mathrm{~B}-\mathrm{H} 3 \mathrm{~B}$

$\mathrm{C} 2 \mathrm{~B}-\mathrm{C} 3 \mathrm{~B}-\mathrm{H} 3 \mathrm{~B}$

$\mathrm{C} 3 \mathrm{~B}-\mathrm{C} 4 \mathrm{~B}-\mathrm{C} 5 \mathrm{~B}$

$\mathrm{C} 3 \mathrm{~B}-\mathrm{C} 4 \mathrm{~B}-\mathrm{H} 4 \mathrm{~B}$

$\mathrm{C} 5 \mathrm{~B}-\mathrm{C} 4 \mathrm{~B}-\mathrm{H} 4 \mathrm{~B}$

$\mathrm{C} 6 \mathrm{~B}-\mathrm{C} 5 \mathrm{~B}-\mathrm{C} 4 \mathrm{~B}$

$\mathrm{C} 6 \mathrm{~B}-\mathrm{C} 5 \mathrm{~B}-\mathrm{H} 5 \mathrm{~B}$

$\mathrm{C} 4 \mathrm{~B}-\mathrm{C} 5 \mathrm{~B}-\mathrm{H} 5 \mathrm{~B}$

$\mathrm{C} 5 \mathrm{~B}-\mathrm{C} 6 \mathrm{~B}-\mathrm{C} 1 \mathrm{~B}$

$\mathrm{C} 5 \mathrm{~B}-\mathrm{C} 6 \mathrm{~B}-\mathrm{H} 6 \mathrm{~B}$

$\mathrm{C} 1 \mathrm{~B}-\mathrm{C} 6 \mathrm{~B}-\mathrm{H} 6 \mathrm{~B}$

$\mathrm{C} 2 \mathrm{C}-\mathrm{C} 1 \mathrm{C}-\mathrm{C} 6 \mathrm{C}$

$\mathrm{C} 2 \mathrm{C}-\mathrm{C} 1 \mathrm{C}-\mathrm{C} 2$

$\mathrm{C} 6 \mathrm{C}-\mathrm{C} 1 \mathrm{C}-\mathrm{C} 2$

$\mathrm{C} 3 \mathrm{C}-\mathrm{C} 2 \mathrm{C}-\mathrm{C} 1 \mathrm{C}$

$\mathrm{C} 3 \mathrm{C}-\mathrm{C} 2 \mathrm{C}-\mathrm{H} 2 \mathrm{C}$

$\mathrm{C} 1 \mathrm{C}-\mathrm{C} 2 \mathrm{C}-\mathrm{H} 2 \mathrm{C}$

$\mathrm{C} 2 \mathrm{C}-\mathrm{C} 3 \mathrm{C}-\mathrm{C} 4 \mathrm{C}$

$\mathrm{C} 2 \mathrm{C}-\mathrm{C} 3 \mathrm{C}-\mathrm{H} 3 \mathrm{C}$

$\mathrm{C} 4 \mathrm{C}-\mathrm{C} 3 \mathrm{C}-\mathrm{H} 3 \mathrm{C}$

$\mathrm{C} 5 \mathrm{C}-\mathrm{C} 4 \mathrm{C}-\mathrm{C} 3 \mathrm{C}$

$\mathrm{C} 5 \mathrm{C}-\mathrm{C} 4 \mathrm{C}-\mathrm{H} 4 \mathrm{C}$

$\mathrm{C} 3 \mathrm{C}-\mathrm{C} 4 \mathrm{C}-\mathrm{H} 4 \mathrm{C}$

$\mathrm{C} 4 \mathrm{C}-\mathrm{C} 5 \mathrm{C}-\mathrm{C} 6 \mathrm{C}$

$\mathrm{C} 4 \mathrm{C}-\mathrm{C} 5 \mathrm{C}-\mathrm{H} 5 \mathrm{C}$

$\mathrm{C} 6 \mathrm{C}-\mathrm{C} 5 \mathrm{C}-\mathrm{H} 5 \mathrm{C}$

$\mathrm{C} 5 \mathrm{C}-\mathrm{C} 6 \mathrm{C}-\mathrm{C} 1 \mathrm{C}$

$\mathrm{C} 5 \mathrm{C}-\mathrm{C} 6 \mathrm{C}-\mathrm{H} 6 \mathrm{C}$

$\mathrm{C} 1 \mathrm{C}-\mathrm{C} 6 \mathrm{C}-\mathrm{H} 6 \mathrm{C}$

$\mathrm{C} 3-\mathrm{O} 3-\mathrm{Ba}$

$\mathrm{C} 3-\mathrm{O} 4-\mathrm{Zn}$

$\mathrm{O} 3-\mathrm{C} 3-\mathrm{O} 4$

$\mathrm{O} 3-\mathrm{C} 3-\mathrm{C} 4$

$\mathrm{O} 4-\mathrm{C} 3-\mathrm{C} 4$

$\mathrm{C} 1 \mathrm{E}-\mathrm{C} 4-\mathrm{C} 1 \mathrm{D}$

$\mathrm{C} 1 \mathrm{E}-\mathrm{C} 4-\mathrm{C} 1 \mathrm{~F}$

$\mathrm{C} 1 \mathrm{D}-\mathrm{C} 4-\mathrm{C} 1 \mathrm{~F}$

$\mathrm{C} 1 \mathrm{E}-\mathrm{C} 4-\mathrm{C} 3$

$\mathrm{C} 1 \mathrm{D}-\mathrm{C} 4-\mathrm{C} 3$

$\mathrm{C} 1 \mathrm{~F}-\mathrm{C} 4-\mathrm{C} 3$

$\mathrm{C} 2 \mathrm{D}-\mathrm{C} 1 \mathrm{D}-\mathrm{C} 6 \mathrm{D}$

$\mathrm{C} 2 \mathrm{D}-\mathrm{C} 1 \mathrm{D}-\mathrm{C} 4$

$\mathrm{C} 6 \mathrm{D}-\mathrm{C} 1 \mathrm{D}-\mathrm{C} 4$

$\mathrm{C} 1 \mathrm{D}-\mathrm{C} 2 \mathrm{D}-\mathrm{C} 3 \mathrm{D}$
$121.2(2)$

119.4

119.4

120.1 (2)

119.9

119.9

$119.8(2)$

120.1

120.1

$119.6(2)$

120.2

120.2

$121.6(2)$

119.2

119.2

118.1 (2)

120.7 (2)

$121.0(2)$

121.4 (2)

119.3

119.3

120.0 (2)

120.0

120.0

119.2 (2)

120.4

120.4

$120.6(2)$

119.7

119.7

120.7 (2)

119.7

119.7

$144.65(16)$

125.09 (16)

$125.0(2)$

$119.3(2)$

$115.6(2)$

$110.1(2)$

111.38 (19)

$107.84(18)$

$105.03(18)$

$110.38(19)$

$112.12(19)$

$117.8(2)$

$122.9(2)$

$119.2(2)$

$120.9(3)$
$\mathrm{O} 2 \mathrm{H}-\mathrm{C} 3 \mathrm{H}-\mathrm{H} 3 \mathrm{H} 2$

$\mathrm{H} 3 \mathrm{H} 1-\mathrm{C} 3 \mathrm{H}-\mathrm{H} 3 \mathrm{H} 2$

$\mathrm{O} 2 \mathrm{H}-\mathrm{C} 3 \mathrm{H}-\mathrm{H} 3 \mathrm{H} 3$

$\mathrm{H} 3 \mathrm{H} 1-\mathrm{C} 3 \mathrm{H}-\mathrm{H} 3 \mathrm{H} 3$

$\mathrm{H} 3 \mathrm{H} 2-\mathrm{C} 3 \mathrm{H}-\mathrm{H} 3 \mathrm{H} 3$

C1I-O1I-Ba

C1I-O1I-H1I

$\mathrm{Ba}-\mathrm{O} 1 \mathrm{I}-\mathrm{H} 1 \mathrm{I}$

$\mathrm{O} 1 \mathrm{I}-\mathrm{C} 1 \mathrm{I}-\mathrm{C} 2 \mathrm{I}$

O1I-C1I-H1I1

$\mathrm{C} 2 \mathrm{I}-\mathrm{C} 1 \mathrm{I}-\mathrm{H} 1 \mathrm{I} 1$

$\mathrm{O} 1 \mathrm{I}-\mathrm{C} 1 \mathrm{I}-\mathrm{H} 1 \mathrm{I} 2$

$\mathrm{C} 2 \mathrm{I}-\mathrm{C} 1 \mathrm{I}-\mathrm{H} 1 \mathrm{I} 2$

$\mathrm{H} 1 \mathrm{I} 1-\mathrm{C} 1 \mathrm{I}-\mathrm{H} 1 \mathrm{I} 2$

$\mathrm{O} 2 \mathrm{I}-\mathrm{C} 2 \mathrm{I}-\mathrm{C} 1 \mathrm{I}$

$\mathrm{O} 2 \mathrm{I}-\mathrm{C} 2 \mathrm{I}-\mathrm{H} 2 \mathrm{I} 1$

$\mathrm{C} 1 \mathrm{I}-\mathrm{C} 2 \mathrm{I}-\mathrm{H} 2 \mathrm{I} 1$

$\mathrm{O} 2 \mathrm{I}-\mathrm{C} 2 \mathrm{I}-\mathrm{H} 2 \mathrm{I} 2$

$\mathrm{C} 1 \mathrm{I}-\mathrm{C} 2 \mathrm{I}-\mathrm{H} 2 \mathrm{I} 2$

$\mathrm{H} 2 \mathrm{I} 1-\mathrm{C} 2 \mathrm{I}-\mathrm{H} 2 \mathrm{I} 2$

$\mathrm{C} 3 \mathrm{I}-\mathrm{O} 2 \mathrm{I}-\mathrm{C} 2 \mathrm{I}$

$\mathrm{O} 2 \mathrm{I}-\mathrm{C} 3 \mathrm{I}-\mathrm{H} 3 \mathrm{I} 1$

$\mathrm{O} 2 \mathrm{I}-\mathrm{C} 3 \mathrm{I}-\mathrm{H} 3 \mathrm{I} 2$

$\mathrm{H} 3 \mathrm{I} 1-\mathrm{C} 3 \mathrm{I}-\mathrm{H} 3 \mathrm{I} 2$

$\mathrm{O} 2 \mathrm{I}-\mathrm{C} 3 \mathrm{I}-\mathrm{H} 3 \mathrm{I} 3$

H3I1-C3I-H3I3

$\mathrm{H} 3 \mathrm{I} 2-\mathrm{C} 3 \mathrm{I}-\mathrm{H} 3 \mathrm{I} 3$

$\mathrm{C} 1 \mathrm{~J}-\mathrm{O} 1 \mathrm{~J}-\mathrm{Ba}$

$\mathrm{C} 1 \mathrm{~J}-\mathrm{O} 1 \mathrm{~J}-\mathrm{H} 1 \mathrm{~J}$

$\mathrm{Ba}-\mathrm{O} 1 \mathrm{~J}-\mathrm{H} 1 \mathrm{~J}$

$\mathrm{O} 1 \mathrm{~J}-\mathrm{C} 1 \mathrm{~J}-\mathrm{C} 2 \mathrm{~J}$

$\mathrm{O} 1 \mathrm{~J}-\mathrm{C} 1 \mathrm{~J}-\mathrm{H} 1 \mathrm{~J} 1$

$\mathrm{C} 2 \mathrm{~J}-\mathrm{C} 1 \mathrm{~J}-\mathrm{H} 1 \mathrm{~J} 1$

$\mathrm{O} 1 \mathrm{~J}-\mathrm{C} 1 \mathrm{~J}-\mathrm{H} 1 \mathrm{~J} 2$

$\mathrm{C} 2 \mathrm{~J}-\mathrm{C} 1 \mathrm{~J}-\mathrm{H} 1 \mathrm{~J} 2$

$\mathrm{H} 1 \mathrm{~J} 1-\mathrm{C} 1 \mathrm{~J}-\mathrm{H} 1 \mathrm{~J} 2$

$\mathrm{O} 2 \mathrm{~J}-\mathrm{C} 2 \mathrm{~J}-\mathrm{C} 1 \mathrm{~J}$

$\mathrm{O} 2 \mathrm{~J}-\mathrm{C} 2 \mathrm{~J}-\mathrm{H} 2 \mathrm{~J} 1$

$\mathrm{C} 1 \mathrm{~J}-\mathrm{C} 2 \mathrm{~J}-\mathrm{H} 2 \mathrm{~J} 1$

$\mathrm{O} 2 \mathrm{~J}-\mathrm{C} 2 \mathrm{~J}-\mathrm{H} 2 \mathrm{~J} 2$

$\mathrm{C} 1 \mathrm{~J}-\mathrm{C} 2 \mathrm{~J}-\mathrm{H} 2 \mathrm{~J} 2$

$\mathrm{H} 2 \mathrm{~J} 1-\mathrm{C} 2 \mathrm{~J}-\mathrm{H} 2 \mathrm{~J} 2$

$\mathrm{C} 3 \mathrm{~J}-\mathrm{O} 2 \mathrm{~J}-\mathrm{C} 2 \mathrm{~J}$

$\mathrm{C} 3 \mathrm{~J}-\mathrm{O} 2 \mathrm{~J}-\mathrm{Ba}$

$\mathrm{C} 2 \mathrm{~J}-\mathrm{O} 2 \mathrm{~J}-\mathrm{Ba}$

$\mathrm{O} 2 \mathrm{~J}-\mathrm{C} 3 \mathrm{~J}-\mathrm{H} 3 \mathrm{~J} 1$

$\mathrm{O} 2 \mathrm{~J}-\mathrm{C} 3 \mathrm{~J}-\mathrm{H} 3 \mathrm{~J} 2$

$\mathrm{H} 3 \mathrm{~J} 1-\mathrm{C} 3 \mathrm{~J}-\mathrm{H} 3 \mathrm{~J} 2$
109.5

109.5

109.5

109.5

109.5

131.91 (16)

103.1

108.4

$111.8(2)$

109.3

109.3

109.3

109.3

107.9

111.8 (2)

109.3

109.3

109.3

109.3

107.9

114.1 (3)

109.5

109.5

109.5

109.5

109.5

109.5

116.81 (15)

105.9

115.3

111.5 (2)

109.3

109.3

109.3

109.3

108.0

108.4 (2)

110.0

110.0

110.0

110.0

108.4

113.4 (2)

124.66 (16)

118.34 (15)

109.5

109.5

109.5 


\begin{tabular}{|c|c|c|c|}
\hline $\mathrm{C} 1 \mathrm{D}-\mathrm{C} 2 \mathrm{D}-\mathrm{H} 2 \mathrm{D}$ & 119.5 & $\mathrm{O} 2 \mathrm{~J}-\mathrm{C} 3 \mathrm{~J}-\mathrm{H} 3 \mathrm{~J} 3$ & 109.5 \\
\hline $\mathrm{C} 3 \mathrm{D}-\mathrm{C} 2 \mathrm{D}-\mathrm{H} 2 \mathrm{D}$ & 119.5 & $\mathrm{H} 3 \mathrm{~J} 1-\mathrm{C} 3 \mathrm{~J}-\mathrm{H} 3 \mathrm{~J} 3$ & 109.5 \\
\hline $\mathrm{C} 4 \mathrm{D}-\mathrm{C} 3 \mathrm{D}-\mathrm{C} 2 \mathrm{D}$ & $120.9(3)$ & $\mathrm{H} 3 \mathrm{~J} 2-\mathrm{C} 3 \mathrm{~J}-\mathrm{H} 3 \mathrm{~J} 3$ & 109.5 \\
\hline $\mathrm{Ba}-\mathrm{O} 1-\mathrm{C} 1-\mathrm{O} 2$ & $31.5(5)$ & $\mathrm{O} 4-\mathrm{C} 3-\mathrm{C} 4-\mathrm{C} 1 \mathrm{D}$ & $178.6(2)$ \\
\hline $\mathrm{Ba}-\mathrm{O} 1-\mathrm{C} 1-\mathrm{C} 2$ & $-151.1(3)$ & $\mathrm{O} 3-\mathrm{C} 3-\mathrm{C} 4-\mathrm{C} 1 \mathrm{~F}$ & $-123.7(2)$ \\
\hline $\mathrm{Zn}-\mathrm{O} 2-\mathrm{C} 1-\mathrm{O} 1$ & $-3.5(3)$ & $\mathrm{O} 4-\mathrm{C} 3-\mathrm{C} 4-\mathrm{C} 1 \mathrm{~F}$ & $58.3(3)$ \\
\hline $\mathrm{Zn}-\mathrm{O} 2-\mathrm{C} 1-\mathrm{C} 2$ & $178.94(14)$ & $\mathrm{C} 1 \mathrm{E}-\mathrm{C} 4-\mathrm{C} 1 \mathrm{D}-\mathrm{C} 2 \mathrm{D}$ & $11.6(3)$ \\
\hline $\mathrm{O} 1-\mathrm{C} 1-\mathrm{C} 2-\mathrm{C} 1 \mathrm{~B}$ & $161.4(2)$ & $\mathrm{C} 1 \mathrm{~F}-\mathrm{C} 4-\mathrm{C} 1 \mathrm{D}-\mathrm{C} 2 \mathrm{D}$ & $-110.1(3)$ \\
\hline $\mathrm{O} 2-\mathrm{C} 1-\mathrm{C} 2-\mathrm{C} 1 \mathrm{~B}$ & $-21.0(3)$ & $\mathrm{C} 3-\mathrm{C} 4-\mathrm{C} 1 \mathrm{D}-\mathrm{C} 2 \mathrm{D}$ & $127.2(2)$ \\
\hline $\mathrm{O} 1-\mathrm{C} 1-\mathrm{C} 2-\mathrm{C} 1 \mathrm{~A}$ & $39.8(3)$ & $\mathrm{C} 1 \mathrm{E}-\mathrm{C} 4-\mathrm{C} 1 \mathrm{D}-\mathrm{C} 6 \mathrm{D}$ & $-173.1(2)$ \\
\hline $\mathrm{O} 2-\mathrm{C} 1-\mathrm{C} 2-\mathrm{C} 1 \mathrm{~A}$ & $-142.6(2)$ & $\mathrm{C} 1 \mathrm{~F}-\mathrm{C} 4-\mathrm{C} 1 \mathrm{D}-\mathrm{C} 6 \mathrm{D}$ & $65.2(3)$ \\
\hline $\mathrm{O} 1-\mathrm{C} 1-\mathrm{C} 2-\mathrm{C} 1 \mathrm{C}$ & $-79.0(3)$ & $\mathrm{C} 3-\mathrm{C} 4-\mathrm{C} 1 \mathrm{D}-\mathrm{C} 6 \mathrm{D}$ & $-57.6(3)$ \\
\hline $\mathrm{O} 2-\mathrm{C} 1-\mathrm{C} 2-\mathrm{C} 1 \mathrm{C}$ & $98.7(2)$ & $\mathrm{C} 6 \mathrm{D}-\mathrm{C} 1 \mathrm{D}-\mathrm{C} 2 \mathrm{D}-\mathrm{C} 3 \mathrm{D}$ & $-0.9(4)$ \\
\hline $\mathrm{C} 1 \mathrm{~B}-\mathrm{C} 2-\mathrm{C} 1 \mathrm{~A}-\mathrm{C} 6 \mathrm{~A}$ & $12.2(3)$ & $\mathrm{C} 4-\mathrm{C} 1 \mathrm{D}-\mathrm{C} 2 \mathrm{D}-\mathrm{C} 3 \mathrm{D}$ & $174.4(2)$ \\
\hline $\mathrm{C} 1 \mathrm{C}-\mathrm{C} 2-\mathrm{C} 1 \mathrm{~A}-\mathrm{C} 6 \mathrm{~A}$ & $-109.0(2)$ & $\mathrm{C} 1 \mathrm{D}-\mathrm{C} 2 \mathrm{D}-\mathrm{C} 3 \mathrm{D}-\mathrm{C} 4 \mathrm{D}$ & $0.8(4)$ \\
\hline $\mathrm{C} 1-\mathrm{C} 2-\mathrm{C} 1 \mathrm{~A}-\mathrm{C} 6 \mathrm{~A}$ & $133.8(2)$ & $\mathrm{C} 2 \mathrm{D}-\mathrm{C} 3 \mathrm{D}-\mathrm{C} 4 \mathrm{D}-\mathrm{C} 5 \mathrm{D}$ & $0.1(4)$ \\
\hline $\mathrm{C} 1 \mathrm{~B}-\mathrm{C} 2-\mathrm{C} 1 \mathrm{~A}-\mathrm{C} 2 \mathrm{~A}$ & $-173.5(2)$ & $\mathrm{C} 3 \mathrm{D}-\mathrm{C} 4 \mathrm{D}-\mathrm{C} 5 \mathrm{D}-\mathrm{C} 6 \mathrm{D}$ & $-0.9(4)$ \\
\hline $\mathrm{C} 1 \mathrm{C}-\mathrm{C} 2-\mathrm{C} 1 \mathrm{~A}-\mathrm{C} 2 \mathrm{~A}$ & $65.3(3)$ & $\mathrm{C} 4 \mathrm{D}-\mathrm{C} 5 \mathrm{D}-\mathrm{C} 6 \mathrm{D}-\mathrm{C} 1 \mathrm{D}$ & $0.8(4)$ \\
\hline $\mathrm{C} 1-\mathrm{C} 2-\mathrm{C} 1 \mathrm{~A}-\mathrm{C} 2 \mathrm{~A}$ & $-51.9(3)$ & $\mathrm{C} 2 \mathrm{D}-\mathrm{C} 1 \mathrm{D}-\mathrm{C} 6 \mathrm{D}-\mathrm{C} 5 \mathrm{D}$ & $0.1(4)$ \\
\hline $\mathrm{C} 6 \mathrm{~A}-\mathrm{C} 1 \mathrm{~A}-\mathrm{C} 2 \mathrm{~A}-\mathrm{C} 3 \mathrm{~A}$ & $-1.0(4)$ & $\mathrm{C} 4-\mathrm{C} 1 \mathrm{D}-\mathrm{C} 6 \mathrm{D}-\mathrm{C} 5 \mathrm{D}$ & $-175.4(2)$ \\
\hline $\mathrm{C} 2-\mathrm{C} 1 \mathrm{~A}-\mathrm{C} 2 \mathrm{~A}-\mathrm{C} 3 \mathrm{~A}$ & $-175.6(2)$ & $\mathrm{C} 1 \mathrm{D}-\mathrm{C} 4-\mathrm{C} 1 \mathrm{E}-\mathrm{C} 2 \mathrm{E}$ & $-103.2(3)$ \\
\hline $\mathrm{C} 1 \mathrm{~A}-\mathrm{C} 2 \mathrm{~A}-\mathrm{C} 3 \mathrm{~A}-\mathrm{C} 4 \mathrm{~A}$ & $-0.3(4)$ & $\mathrm{C} 1 \mathrm{~F}-\mathrm{C} 4-\mathrm{C} 1 \mathrm{E}-\mathrm{C} 2 \mathrm{E}$ & $16.4(3)$ \\
\hline $\mathrm{C} 2 \mathrm{~A}-\mathrm{C} 3 \mathrm{~A}-\mathrm{C} 4 \mathrm{~A}-\mathrm{C} 5 \mathrm{~A}$ & $0.9(4)$ & $\mathrm{C} 3-\mathrm{C} 4-\mathrm{C} 1 \mathrm{E}-\mathrm{C} 2 \mathrm{E}$ & $138.0(2)$ \\
\hline $\mathrm{C} 3 \mathrm{~A}-\mathrm{C} 4 \mathrm{~A}-\mathrm{C} 5 \mathrm{~A}-\mathrm{C} 6 \mathrm{~A}$ & $-0.1(4)$ & $\mathrm{C} 1 \mathrm{D}-\mathrm{C} 4-\mathrm{C} 1 \mathrm{E}-\mathrm{C} 6 \mathrm{E}$ & $74.1(3)$ \\
\hline $\mathrm{C} 4 \mathrm{~A}-\mathrm{C} 5 \mathrm{~A}-\mathrm{C} 6 \mathrm{~A}-\mathrm{C} 1 \mathrm{~A}$ & $-1.3(4)$ & $\mathrm{C} 1 \mathrm{~F}-\mathrm{C} 4-\mathrm{C} 1 \mathrm{E}-\mathrm{C} 6 \mathrm{E}$ & $-166.3(2)$ \\
\hline $\mathrm{C} 2 \mathrm{~A}-\mathrm{C} 1 \mathrm{~A}-\mathrm{C} 6 \mathrm{~A}-\mathrm{C} 5 \mathrm{~A}$ & $1.8(4)$ & $\mathrm{C} 3-\mathrm{C} 4-\mathrm{C} 1 \mathrm{E}-\mathrm{C} 6 \mathrm{E}$ & $-44.7(3)$ \\
\hline $\mathrm{C} 2-\mathrm{C} 1 \mathrm{~A}-\mathrm{C} 6 \mathrm{~A}-\mathrm{C} 5 \mathrm{~A}$ & $176.3(2)$ & $\mathrm{C} 6 \mathrm{E}-\mathrm{C} 1 \mathrm{E}-\mathrm{C} 2 \mathrm{E}-\mathrm{C} 3 \mathrm{E}$ & $-0.9(4)$ \\
\hline $\mathrm{C} 1 \mathrm{~A}-\mathrm{C} 2-\mathrm{C} 1 \mathrm{~B}-\mathrm{C} 2 \mathrm{~B}$ & $-108.0(2)$ & $\mathrm{C} 4-\mathrm{C} 1 \mathrm{E}-\mathrm{C} 2 \mathrm{E}-\mathrm{C} 3 \mathrm{E}$ & $176.4(2)$ \\
\hline $\mathrm{C} 1 \mathrm{C}-\mathrm{C} 2-\mathrm{C} 1 \mathrm{~B}-\mathrm{C} 2 \mathrm{~B}$ & $12.0(3)$ & $\mathrm{C} 1 \mathrm{E}-\mathrm{C} 2 \mathrm{E}-\mathrm{C} 3 \mathrm{E}-\mathrm{C} 4 \mathrm{E}$ & $0.3(4)$ \\
\hline $\mathrm{C} 1-\mathrm{C} 2-\mathrm{C} 1 \mathrm{~B}-\mathrm{C} 2 \mathrm{~B}$ & $128.7(2)$ & $\mathrm{C} 2 \mathrm{E}-\mathrm{C} 3 \mathrm{E}-\mathrm{C} 4 \mathrm{E}-\mathrm{C} 5 \mathrm{E}$ & $0.6(4)$ \\
\hline $\mathrm{C} 1 \mathrm{~A}-\mathrm{C} 2-\mathrm{C} 1 \mathrm{~B}-\mathrm{C} 6 \mathrm{~B}$ & $67.2(3)$ & $\mathrm{C} 3 \mathrm{E}-\mathrm{C} 4 \mathrm{E}-\mathrm{C} 5 \mathrm{E}-\mathrm{C} 6 \mathrm{E}$ & $-0.8(4)$ \\
\hline $\mathrm{C} 1 \mathrm{C}-\mathrm{C} 2-\mathrm{C} 1 \mathrm{~B}-\mathrm{C} 6 \mathrm{~B}$ & $-172.85(19)$ & $\mathrm{C} 4 \mathrm{E}-\mathrm{C} 5 \mathrm{E}-\mathrm{C} 6 \mathrm{E}-\mathrm{C} 1 \mathrm{E}$ & $0.1(4)$ \\
\hline $\mathrm{C} 1-\mathrm{C} 2-\mathrm{C} 1 \mathrm{~B}-\mathrm{C} 6 \mathrm{~B}$ & $-56.1(3)$ & $\mathrm{C} 2 \mathrm{E}-\mathrm{C} 1 \mathrm{E}-\mathrm{C} 6 \mathrm{E}-\mathrm{C} 5 \mathrm{E}$ & $0.7(4)$ \\
\hline $\mathrm{C} 6 \mathrm{~B}-\mathrm{C} 1 \mathrm{~B}-\mathrm{C} 2 \mathrm{~B}-\mathrm{C} 3 \mathrm{~B}$ & $-0.9(3)$ & $\mathrm{C} 4-\mathrm{C} 1 \mathrm{E}-\mathrm{C} 6 \mathrm{E}-\mathrm{C} 5 \mathrm{E}$ & $-176.7(2)$ \\
\hline $\mathrm{C} 2-\mathrm{C} 1 \mathrm{~B}-\mathrm{C} 2 \mathrm{~B}-\mathrm{C} 3 \mathrm{~B}$ & $174.4(2)$ & $\mathrm{C} 1 \mathrm{E}-\mathrm{C} 4-\mathrm{C} 1 \mathrm{~F}-\mathrm{C} 6 \mathrm{~F}$ & $92.9(3)$ \\
\hline $\mathrm{C} 1 \mathrm{~B}-\mathrm{C} 2 \mathrm{~B}-\mathrm{C} 3 \mathrm{~B}-\mathrm{C} 4 \mathrm{~B}$ & $0.0(4)$ & $\mathrm{C} 1 \mathrm{D}-\mathrm{C} 4-\mathrm{C} 1 \mathrm{~F}-\mathrm{C} 6 \mathrm{~F}$ & $-146.2(3)$ \\
\hline $\mathrm{C} 2 \mathrm{~B}-\mathrm{C} 3 \mathrm{~B}-\mathrm{C} 4 \mathrm{~B}-\mathrm{C} 5 \mathrm{~B}$ & $0.6(4)$ & $\mathrm{C} 3-\mathrm{C} 4-\mathrm{C} 1 \mathrm{~F}-\mathrm{C} 6 \mathrm{~F}$ & $-24.4(3)$ \\
\hline $\mathrm{C} 3 \mathrm{~B}-\mathrm{C} 4 \mathrm{~B}-\mathrm{C} 5 \mathrm{~B}-\mathrm{C} 6 \mathrm{~B}$ & $-0.3(4)$ & $\mathrm{C} 1 \mathrm{E}-\mathrm{C} 4-\mathrm{C} 1 \mathrm{~F}-\mathrm{C} 2 \mathrm{~F}$ & $-84.8(3)$ \\
\hline $\mathrm{C} 4 \mathrm{~B}-\mathrm{C} 5 \mathrm{~B}-\mathrm{C} 6 \mathrm{~B}-\mathrm{C} 1 \mathrm{~B}$ & $-0.6(4)$ & $\mathrm{C} 1 \mathrm{D}-\mathrm{C} 4-\mathrm{C} 1 \mathrm{~F}-\mathrm{C} 2 \mathrm{~F}$ & $36.1(3)$ \\
\hline $\mathrm{C} 2 \mathrm{~B}-\mathrm{C} 1 \mathrm{~B}-\mathrm{C} 6 \mathrm{~B}-\mathrm{C} 5 \mathrm{~B}$ & $1.1(3)$ & $\mathrm{C} 3-\mathrm{C} 4-\mathrm{C} 1 \mathrm{~F}-\mathrm{C} 2 \mathrm{~F}$ & $157.8(2)$ \\
\hline $\mathrm{C} 2-\mathrm{C} 1 \mathrm{~B}-\mathrm{C} 6 \mathrm{~B}-\mathrm{C} 5 \mathrm{~B}$ & $-174.3(2)$ & $\mathrm{C} 6 \mathrm{~F}-\mathrm{C} 1 \mathrm{~F}-\mathrm{C} 2 \mathrm{~F}-\mathrm{C} 3 \mathrm{~F}$ & $-1.6(4)$ \\
\hline $\mathrm{C} 1 \mathrm{~B}-\mathrm{C} 2-\mathrm{C} 1 \mathrm{C}-\mathrm{C} 2 \mathrm{C}$ & $-89.5(3)$ & $\mathrm{C} 4-\mathrm{C} 1 \mathrm{~F}-\mathrm{C} 2 \mathrm{~F}-\mathrm{C} 3 \mathrm{~F}$ & $176.3(2)$ \\
\hline $\mathrm{C} 1 \mathrm{~A}-\mathrm{C} 2-\mathrm{C} 1 \mathrm{C}-\mathrm{C} 2 \mathrm{C}$ & $30.8(3)$ & $\mathrm{C} 1 \mathrm{~F}-\mathrm{C} 2 \mathrm{~F}-\mathrm{C} 3 \mathrm{~F}-\mathrm{C} 4 \mathrm{~F}$ & $-0.1(4)$ \\
\hline $\mathrm{C} 1-\mathrm{C} 2-\mathrm{C} 1 \mathrm{C}-\mathrm{C} 2 \mathrm{C}$ & $151.7(2)$ & $\mathrm{C} 2 \mathrm{~F}-\mathrm{C} 3 \mathrm{~F}-\mathrm{C} 4 \mathrm{~F}-\mathrm{C} 5 \mathrm{~F}$ & $1.8(5)$ \\
\hline $\mathrm{C} 1 \mathrm{~B}-\mathrm{C} 2-\mathrm{C} 1 \mathrm{C}-\mathrm{C} 6 \mathrm{C}$ & $87.1(3)$ & $\mathrm{C} 3 \mathrm{~F}-\mathrm{C} 4 \mathrm{~F}-\mathrm{C} 5 \mathrm{~F}-\mathrm{C} 6 \mathrm{~F}$ & $-1.9(5)$ \\
\hline $\mathrm{C} 1 \mathrm{~A}-\mathrm{C} 2-\mathrm{C} 1 \mathrm{C}-\mathrm{C} 6 \mathrm{C}$ & $-152.6(2)$ & $\mathrm{C} 2 \mathrm{~F}-\mathrm{C} 1 \mathrm{~F}-\mathrm{C} 6 \mathrm{~F}-\mathrm{C} 5 \mathrm{~F}$ & $1.5(4)$ \\
\hline $\mathrm{C} 1-\mathrm{C} 2-\mathrm{C} 1 \mathrm{C}-\mathrm{C} 6 \mathrm{C}$ & $-31.7(3)$ & $\mathrm{C} 4-\mathrm{C} 1 \mathrm{~F}-\mathrm{C} 6 \mathrm{~F}-\mathrm{C} 5 \mathrm{~F}$ & $-176.2(3)$ \\
\hline
\end{tabular}




\begin{tabular}{ll}
$\mathrm{C} 6 \mathrm{C}-\mathrm{C} 1 \mathrm{C}-\mathrm{C} 2 \mathrm{C}-\mathrm{C} 3 \mathrm{C}$ & $-1.3(4)$ \\
$\mathrm{C} 2-\mathrm{C} 1 \mathrm{C}-\mathrm{C} 2 \mathrm{C}-\mathrm{C} 3 \mathrm{C}$ & $175.4(2)$ \\
$\mathrm{C} 1 \mathrm{C}-\mathrm{C} 2 \mathrm{C}-\mathrm{C} 3 \mathrm{C}-\mathrm{C} 4 \mathrm{C}$ & $0.4(4)$ \\
$\mathrm{C} 2 \mathrm{C}-\mathrm{C} 3 \mathrm{C}-\mathrm{C} 4 \mathrm{C}-\mathrm{C} 5 \mathrm{C}$ & $0.5(4)$ \\
$\mathrm{C} 3 \mathrm{C}-\mathrm{C} 4 \mathrm{C}-\mathrm{C} 5 \mathrm{C}-\mathrm{C} 6 \mathrm{C}$ & $-0.5(4)$ \\
$\mathrm{C} 4 \mathrm{C}-\mathrm{C} 5 \mathrm{C}-\mathrm{C} 6 \mathrm{C}-\mathrm{C} 1 \mathrm{C}$ & $-0.4(4)$ \\
$\mathrm{C} 2 \mathrm{C}-\mathrm{C} 1 \mathrm{C}-\mathrm{C} 6 \mathrm{C}-\mathrm{C} 5 \mathrm{C}$ & $1.2(4)$ \\
$\mathrm{C} 2-\mathrm{C} 1 \mathrm{C}-\mathrm{C} 6 \mathrm{C}-\mathrm{C} 5 \mathrm{C}$ & $-175.4(2)$ \\
$\mathrm{Ba}-\mathrm{O} 3-\mathrm{C} 3-\mathrm{O} 4$ & $13.4(5)$ \\
$\mathrm{Ba}-\mathrm{O} 3-\mathrm{C} 3-\mathrm{C} 4$ & $-164.39(19)$ \\
$\mathrm{Zn}-\mathrm{O} 4-\mathrm{C} 3-\mathrm{O} 3$ & $22.2(3)$ \\
$\mathrm{Zn}-\mathrm{O} 4-\mathrm{C} 3-\mathrm{C} 4$ & $-159.98(15)$ \\
$\mathrm{O} 3-\mathrm{C} 3-\mathrm{C} 4-\mathrm{C} 1 \mathrm{E}$ & $115.2(2)$ \\
$\mathrm{O} 4-\mathrm{C} 3-\mathrm{C} 4-\mathrm{C} 1 \mathrm{E}$ & $-62.8(3)$ \\
$\mathrm{O} 3-\mathrm{C} 3-\mathrm{C} 4-\mathrm{C} 1 \mathrm{D}$ & $-3.4(3)$ \\
\hline
\end{tabular}

$0.2(5)$

$-8.8(3)$

$54.1(3)$

$71.7(3)$

$-67.0(2)$

$-4.1(7)$

72.7 (4)

$174.4(2)$

$-140.4(2)$

$-63.6(3)$

$105.6(3)$

$-46.1(3)$

$56.2(3)$

$159.6(2)$

$-40.8(3)$

Hydrogen-bond geometry $\left(A,{ }^{\circ}\right)$

\begin{tabular}{lllll}
\hline$D-\mathrm{H} \cdots A$ & $D-\mathrm{H}$ & $\mathrm{H} \cdots A$ & $D \cdots A$ & $D-\mathrm{H} \cdots A$ \\
\hline $\mathrm{O} 1 G-\mathrm{H} 1 G \cdots \mathrm{O} 2 I^{\mathrm{i}}$ & 0.84 & 1.91 & $2.728(3)$ & 163 \\
$\mathrm{O} 1 H-\mathrm{H} 1 H \cdots \mathrm{O} 3$ & 0.84 & 2.17 & $2.746(3)$ & 125 \\
$\mathrm{O} 1 I-\mathrm{H} 1 I \cdots \mathrm{O} 2 H$ & 0.84 & 1.99 & $2.817(3)$ & 170 \\
$\mathrm{C} 2 I-\mathrm{H} 2 I 2 \cdots \mathrm{C} 11^{\mathrm{ii}}$ & 0.99 & 2.81 & $3.660(3)$ & 144 \\
$\mathrm{O} 1 J-\mathrm{H} 1 J \cdots \mathrm{Cl} 22^{\mathrm{iii}}$ & 0.84 & 2.17 & $3.012(2)$ & 174
\end{tabular}

Symmetry codes: (i) $x-1, y, z$; (ii) $x+1, y, z$; (iii) $x, y+1, z$. 